\title{
A Microeconomic Explanation of the EPK Paradox
}

\author{
Wolfgang Härdle* \\ Volker Krätschmer** \\ Rouslan Moro***
}

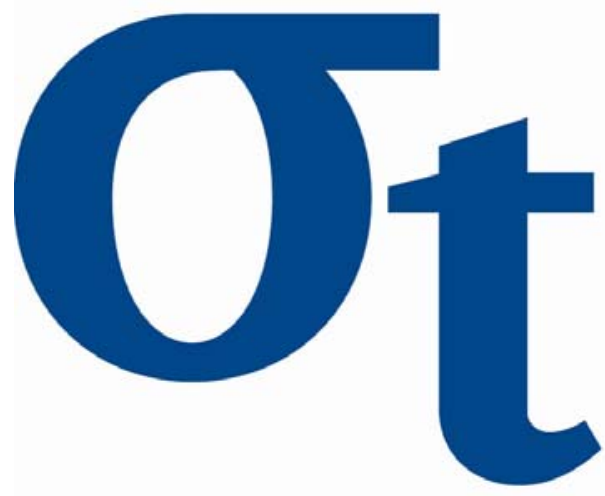

This research was supported by the Deutsche Forschungsgemeinschaft through the SFB 649 "Economic Risk". 


\title{
A Microeconomic Explanation of the EPK Paradox*
}

\author{
Wolfgang K. Härdle† Volker Krätschmer; Rouslan A. Moro§
}

\begin{abstract}
Supported by several recent investigations the empirical pricing kernel paradox might be considered as a stylized fact. In Chabi-Yo et al. (2008) simulation studies have been presented which suggest that this paradox might be caused by regime switching of stock prices in financial markets. Alternatively, we want to emphasize a microeconomic view. Based on an economic model with state dependent utilities for the financial investors we succeed in explaining the paradox by changes of the risk attitudes. Theoretically, the change behaviour is compressed by the pricing kernels. As a starting point for empirical insights we shall develop and investigate inverse problems in terms of data fits for estimated basic values of the pricing kernel.
\end{abstract}

KEYWORDS: Pricing kernel, representative agent, empirical pricing kernel, epk paradox, state dependent utilities, switching points. JEL Classification: D01, D58, C02, G13

AMS Classification: 15A29, 62G07, 62G35

*This research was supported by Deutsche Forschungsgemeinschaft through the SFB 649 "Economic Risk".

${ }^{\dagger}$ Center for Applied Statistics and Economics, Humboldt-Universität zu Berlin, Spandauer Str. 1, 10178 Berlin; e-mail: haerdle@wiwi.hu-berlin.de.

${ }^{\ddagger}$ Center for Applied Statistics and Economics, Humboldt-Universität zu Berlin, Spandauer Str. 1, 10178 Berlin and Institute of Mathematics, Berlin University of Technology, 10623 Berlin; e-mail: kraetsch@math.tu-berlin.de.

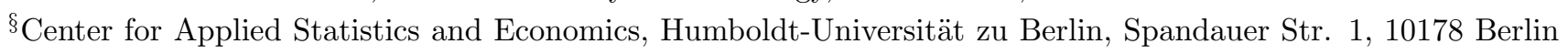
and German Institute for Economic Research (DIW Berlin), Mohrenstr. 58, 10117 Berlin; e-mail: moro@diw.de. 


\section{Introduction}

The empirical pricing kernel paradox refers to the empirical phenomenon that the observable behaviour of investors in financial markets conflicts with the traditional expected utility framework. Several recent studies support the conjecture that this deviation evolves into a stylized fact. All the investigations are settled within similar economic models assuming representative agents in financial markets whose indirect preferences have classical expected utility representation. Additionally, the risk neutral valuation principle is supposed to be valid for the financial markets by means of pricing kernels. If the pricing kernels represent state contingent equilibrium prices they might be identified with the v. Neumann-Morgenstern indices of the representative agents. As a consequence the pricing kernels should be nonincreasing.

In the studies of Ait-Sahalia and Lo (2000), Jackwerth (2000), Detlefsen et al. (2007), Constantinidis et al. (2009) different econometric methods have been applied to estimate pricing kernels with varying underlying models for the financial markets. It turned out as a common result, that the estimates, the so called empirical pricing kernels, have non-monotonic shape regardless of the used data sets. This difference between the theoretical property of the pricing kernel and the observed failure of it is what we shall call the empirical pricing kernel paradox. A further confirmation of it is provided by Golubev et al. (2008). In this paper a test for monotonicity of pricing kernels has been introduced, and applied to DAX data over several periods. Typically, the nullhypothesis that the pricing kernel is nonincreasing was rejected.

More recently, simulation studies in Chabi-Yo et al. (2008) suggest to explain by regime switches of the prices for the underlyings of the financial markets. Since it is plausible to view regime switches as consequences of changes in the investor' risk attitude, we want to emphasize a microeconomic perspective. The aim is to provide an extended economic model, which allows non-monotone pricing kernels. The crucial idea is to propose financial investors whose risk attidudes might be sensible to the prices in the financial markets. More technically, we shall assume state dependent utilities to represent the preferences of the financial investors.

The paper is organized as follows. In section2 we shall introduce our model for the financial market. Also the classical relationship between the utilities of representative agent and the pricing kernel will be reviewed. Afterwards, the problem to estimate pricing kernel will be considered. Afterwards we shall point out the empirical pricing kernel paradox. A simple consumption model based on state dependent utilities 
for the investors will be introduced in section 3. Within this framework we shall retain the relationship between preferences of investors and pricing in the market by an analogous result. It will turn out that dependent on the stock prices the v. Neumann-Morgenstern utility index for the representative agent might switch between different types of utilities, meaning possible changes of the risk attitudes. In particular pricing kernels might be non-monotone. The switching points and the types of utilities describe local risk attitudes of the investors. So in order to get some insight on the changing of the risk attitudes it might be useful to analyze the switching behaviour of the pricing kernel. The idea is to find a good fit of estimated basic values by given base functions expressing the possible types utilities. Essentially, there are inverse problems behind which will be developed and investigated throughout section 4 . Several mathematical results and proofs of quite technical nature have been delegated to appendices A, B, C respectively.

\section{Financial Investors' within the Expected Utility Frame- work and the Empirical Pricing Kernel Paradox}

Let $[0, T]$ be the time interval of investment in the financial market, where $t=0$ denotes the present time and $t=T \in] 0, \infty[$ the time of maturity.

Furthermore it is assumed that a riskless bond and a risky asset are traded in the financial market as basic underlyings. The price process $\left(B_{t}\right)_{t \in[0, T]}$ of the riskless bond is defined by

$$
\frac{d B_{t}}{B_{t}}=r_{t} d t
$$

via a deterministic Riemannian-integrable interest process $\left(r_{t}\right)_{t \in[0, T]}$. The price process $\left(S_{t}\right)_{t \in[0, T]}$ of the risky asset is assumed to be a nonnegative semimartingale with constant $S_{0}$ and continuously distributed marginals $\left.\left.S_{t}(t \in] 0, T\right]\right)$. Outstanding examples for such financial markets are the Black-Scholes model, non-parametric diffusion models as in Ait-Sahalia and Lo (2000) and GARCH models. Notice that time discrete models may be subsumed under this setting.

Furthermore let us suppose that the financial market is arbitrage free in the sense that there exists at least one equivalent martingale measure.

We further assume that the risk neutral valuation principle is valid for nonnegative pay offs $\psi\left(S_{T}\right)$. That means that there is some unknown Radon-Nikodym density $\pi$ of a martingale measure such that the price 
of any $\psi\left(S_{T}\right)$ is characterized by

$$
\mathbb{E}\left[e^{-\int_{0}^{T} r_{x} d x} \psi\left(S_{T}\right) \pi\right]=\mathbb{E}\left[e^{-\int_{0}^{T} r_{x} d x} \psi\left(S_{T}\right) \mathbb{E}\left[\pi \mid S_{T}\right]\right] .
$$

By factorization we find some Borel-measurable $\mathcal{K}_{\pi}$ with $\mathbb{E}\left[\pi \mid S_{T}\right]=\mathcal{K}_{\pi}\left(S_{T}\right)$, so that

$$
\mathbb{E}\left[e^{-\int_{0}^{T} r_{x} d x} \psi\left(S_{T}\right) \pi\right]=\int_{0}^{\infty} e^{-\int_{0}^{T} r_{x} d x} \psi(x) \mathcal{K}_{\pi}(x) p_{S_{T}}(x) d x
$$

where $p_{S_{T}}$ denotes a density function of the distribution of $S_{T}$. Equation (2) gives reason to call $\mathcal{K}_{\pi}$ the pricing kernel (w.r.t. $\pi$ ).

Let us now embed the financial market into an economic model where the investors of the financial market are consumers whose consumptions rely on the price $S_{T}$ of the stock at maturity only. Within the classical framework, where the investor preferences may be represented by expected utilities, there exists a link between the risk attitude of the investors and the pricing rule of the financial markets. It is built upon the assumption of a representative agent whose indirect utility $U\left(\left\{\bar{e}\left(S_{T}\right)\right\}\right)$, depending on the aggregated market endowment $\bar{e}\left(S_{T}\right)$, has expected utility representation $U\left(\bar{e}\left[S_{T}\right]\right)=\mathbb{E}\left[u\left(S_{T}\right)\right]$ with concave v. Neumann-Morgenstern utility index $u$. Under some further technical conditions on the investor preferences, and the additional specification $\bar{e}\left(S_{T}\right)=\frac{S_{T}}{S_{0}}$, then there is some positive $\beta$ such that

$$
\left.\frac{d u}{d x}\right|_{x=\frac{s_{T}}{S_{0}}}=\beta \mathcal{K}_{\pi}\left(\frac{s_{T}}{S_{0}}\right)
$$

for almost every realization $s_{T}$ of $S_{T}$. This relationship might be obtained by using methods as in the sections 6.1, 6.2 of Karatzas and Shreve (1998). For a rigorous formulation and derivation see Corollary A.2 in Appendix A. It should be emphasized that within the classical expected utility framework the pricing kernel has to be nonincreasing due to concavity of the utility index $u$.

Several recent econometric studies are concerned with the problem to estimate the pricing kernel, calling the estimators empirical pricing kernels (EPK) (cf. Ait-Sahalia and Lo (2000), Jackwerth (2000), Detlefsen et al. (2007), Constantinidis et al. (2009)). Typically, we may find a shape of the empirical pricing kernel as visualized in the following graphic which is borrowed from Detlefsen et al. (2007).

The figure shows empirical pricing kernels resulting from different estimation methods. Here estimations of Garch-, Garch-M and discrete Heston models for stock prices had been done. The underlying data are the from the DAX index in March 2000. Significantly, all three kernels are quite similar: They have the 


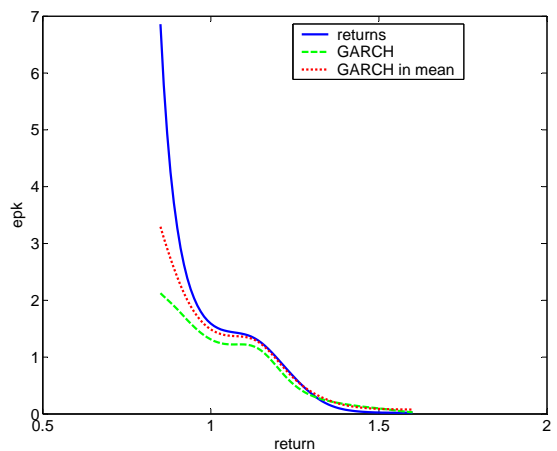

Figure 1: EPK on 24 March 2000. Across data sets, methods, models and markets

same form, the same characteristic features like e.g. the hump, and they differ in absolute terms slightly. In particular the empirical kernels fail to be monotone, contrasting the classical theory within the expected utility framework. This is what we shall call the empirical pricing kernel paradox.

A further investigation in Detlefsen et al. (2007) based on DAX data in July 2002 and June 2004 confirmed the paradox. Moreover, in the above mentioned studies Ait-Sahalia and Lo (2000), Jackwerth (2000) one may find similar pictures of the empirical pricing kernels. So summarizing, the empirical pricing kernel paradox has been observed across different times independently of the data sets, the markets, the models of stock prices and the employed estimation methods. It is further supported by Golubev et al. (2008), where a statistical test for the monotonicity of pricing kernel has been developed. The application to DAX data over different periods lead to the rejection of the null hypothesis that the pricing kernel is nondecreasing, in almost every case.

\section{A Microeconomic View on the EPK Paradox}

A first explanation for the empirical pricing kernel paradox has been offered by Chabi-Yo et al. (2008). The crucial idea of the authors is to suppose that regime switches are inherent of the price process of the stock market. More specifially, within a discrete time period $\{0,1, \ldots, T\}$, there are two types of price processes $\left(S_{t}^{0}\right)_{t \in\{0, \ldots, T\}},\left(S_{t}^{1}\right)_{t \in\{0, \ldots, T\}}$ for the risky asset which have joint continuous distributions, and constitute separately together with the riskless bond arbitrage free financial markets in the sense of section 2. Furthermore, Chabi-Yo et al. (2008) assume a latent regime switching variables in terms of an 
unobservable Markov-chain $\left(U_{t}\right)_{t \in\{0,1, \ldots, T\}}$ of Bernoulli-distributed random variables. The observable price process $\left(S_{t}\right)_{t \in\{0,1, \ldots, T\}}$ is then modelled by $S_{t}=U_{t} S_{t}^{1}+\left(1-U_{t}\right) S_{t}^{0}$ for $t \in\{0, \ldots, T\}$. Assuming the risk neutral valuation principle for the latent two basic financial markets and for the observable one, the authors drew a comparison of the associated pricing kernels via a simulation study. Indeed it turned out that the empirical pricing kernels in the separated financial market were nondecreasing whereas the empirical pricing kernel in the integrated financial market failed to have the property of monotonicity. Therefore the empirical pricing kernel might be explained by a switch of the price processes of the underlyings in the financial market.

Referring to Chabi-Yo et al. (2008), we want to stress a microeconomic viewpoint. Based on the initializing thought that regime switching is caused by changes of the investors' preferences our aim is to make the influence of these changes on the shape of the pricing kernels more explicit. In the following we shall provide a simple economic model underlying the financial market where the pricing kernel need not to be nonincreasing. The key idea is to consider the investors as consumers whose preferences are representable by utilities dependent on the prices of the stock. This refers to the concept of state dependent preferences. An axiomatic justification is provided by Karni et al. (1983).

Let us assume that we have $m$ consumers who choose among nonnegative random variables $c\left(S_{T}\right)$. They have exogeneous endowments by initial capitals $w_{0}^{1}, \ldots, w_{0}^{m}>0$ and state dependent endowement in form of nonnegative random variables $e_{1}\left(S_{T}\right), \ldots, e_{m}\left(S_{T}\right)$. Their individual budget constraints for $c\left(S_{T}\right)$ is therefore:

$$
\int_{0}^{\infty} \exp \left(-\int_{0}^{T} r_{y} d y\right) c(x) \mathcal{K}_{\pi}(x) p_{S_{T}}(x) d x \leq w_{0}^{i}+\int_{0}^{\infty} \exp \left(-\int_{0}^{T} r_{y} d y\right) e_{i}(x) \mathcal{K}_{\pi}(x) p_{S_{T}}(x) d x, i=1, \ldots, m .
$$

The consumers are assumed to have state dependent utilities in terms of extended expected utility preferences within the terminology of Mas-Colell et al. (1995). That means in particular that consumer $i$ has numerical representation of her/his preferences as:

$$
U^{i}\left\{c\left(S_{T}\right)\right\}=\mathbb{E}\left[\exp \left(-\int_{0}^{T} r_{x} d x\right) u^{i}\left\{S_{T}, c\left(S_{T}\right)\right\}\right]
$$

where $u^{i}: \mathbb{R}_{+} \times \mathbb{R}_{+} \rightarrow \mathbb{R} \cup\{-\infty\}$ denotes a state dependent v. Neumann-Morgenstern utility index 
satisfying:

$$
\begin{aligned}
& u^{i}(x, y) \in \mathbb{R} \text { for } x \geq 0, \quad y>0, \\
& u^{i}(x, \cdot) \quad \text { is strictly increasing and strictly concave for any } x \geq 0, \\
& u^{i}(\cdot, y) \quad \text { is Borel-measurable for every } y \geq 0 .
\end{aligned}
$$

At time of maturity $T$ the market has an aggregated endowment $\bar{e}\left(S_{T}\right) \stackrel{\text { def }}{=} \sum_{i=1}^{m}\left\{w_{0}^{i}+e_{i}\left(S_{T}\right)\right\}$. It is assumed that simultaneous consumption is allowed for consumption vectors $\left(c_{1}\left(S_{T}\right), \ldots, c_{m}\left(S_{T}\right)\right)$ which satisfy the individual budget constraints and obey the aggregated endowment in the sense of $\sum_{i=1}^{m} c_{i}\left(S_{T}\right) \leq \bar{e}\left(S_{T}\right)$. Such vectors are called admissible, and they are gathered by a set say $\mathcal{A}$ (see Mas-Colell et al. (1995)).

Next it is supposed that the consumers have chosen their consumptions $\left(\bar{c}_{1}\left(S_{T}\right), \ldots, \bar{c}_{m}\left(S_{T}\right)\right)$ such that the following properties are fulfilled.

(ii) individual optimization: For each consumer $i$ the consumption $\bar{c}_{i}\left(S_{T}\right)$ solves the optimization problem

$$
\begin{aligned}
& \max U^{i}\left\{c\left(S_{T}\right)\right\} \\
& \text { s.t. } c\left(S_{T}\right) \text { satisfies individual budget constraint (3). }
\end{aligned}
$$

(i) market clearing:

$$
\sum_{i=1}^{m} \bar{c}_{i}\left(S_{T}\right)=\bar{e}\left(S_{T}\right)
$$

The conditions (8) and (8) describe a weak version of a so called contingent Arrow Debreu equilibrium (see Dana and Jeanblanc (2003), sect. 7.1). As a by product $\left(\bar{c}_{1}\left(S_{T}\right), \ldots, \bar{c}_{m}\left(S_{T}\right)\right.$ ) is a Pareto optimum too, i.e. there is no $\left(c_{1}\left(S_{T}\right), \ldots, c_{m}\left(S_{T}\right)\right) \in \mathcal{A}$ with $U^{i}\left\{c_{i}\left(S_{T}\right)\right\} \geq U^{i}\left\{\bar{c}_{i}\left(S_{T}\right)\right\}$ for every $i$ and such that $U^{i_{0}}\left\{c_{i_{0}}\left(S_{T}\right)\right\}>U^{i_{0}}\left\{\bar{c}_{i_{0}}\left(S_{T}\right)\right\}$ for at least one $i_{0}$. Therefore, by the so called Negeishi method (cf. Dana and Jeanblanc (2003)) we may find nonnegative weights $\alpha_{1}, \ldots, \alpha_{m}$ summing to 1 such that

$$
\sum_{i=1}^{m} \alpha_{i} U^{i}\left\{\bar{c}^{i}\left(S_{T}\right)\right\}=U_{\alpha}\left\{\bar{e}\left(S_{T}\right)\right\} \stackrel{\text { def }}{=} \max \left\{\sum_{i=1}^{m} \alpha_{i} U^{i}\left\{c_{i}\left(S_{T}\right)\right\} \mid \sum_{i=1}^{m} c_{i}\left(S_{T}\right) \leq \bar{e}\left(S_{T}\right)\right\}
$$

Let $u_{\alpha}: \mathbb{R}_{+}^{2} \rightarrow \mathbb{R} \cup\{-\infty, \infty\}$ be defined by

$$
u_{\alpha}(x, y)=\sup \left\{\exp \left(-\int_{0}^{T} r_{x} d x\right) \sum_{i=1}^{m} \alpha_{i} u_{1}^{i}\left(x, y_{i}\right) \mid y_{1}, \ldots, y_{m} \geq 0, \sum_{i=1}^{m} y_{i} \leq y\right\} .
$$


Obviously, $u_{\alpha}(x, \cdot)$ is strictly increasing as well as strictly concave for $x \geq 0$, and $u_{\alpha}(\cdot, y)$ is Borel-measurable for every $y \geq 0$.

$U_{\alpha}\left\{\bar{e}\left(S_{T}\right)\right\}$ has extended expected utility representation

$$
U_{\alpha}\left\{\bar{e}\left(S_{T}\right)\right\}=\mathbb{E}\left[\exp \left(-\int_{0}^{T} r_{x} d x\right) u_{\alpha}\left(\bar{e}\left(S_{T}\right)\right)\right],
$$

which might be concluded from Lemmata B.1, B.2 (cf. Appendix B). In the next step we want to establish the relationship between the indirect utility $u_{\alpha}$ of the representative agent and the pricing kernel $\mathcal{K}_{\pi}$. As customary, we shall impose the so called Inada conditions (cf. Dana and Jeanblanc (2003)) on the state dependent utility indices $u^{1}\left(S_{T}, \cdot\right), \ldots, u^{m}\left(S_{T}, \cdot\right)$, i.e. $\left.u^{1}(x, \cdot) \mid\right] 0, \infty\left[, \ldots, u^{m}(x, \cdot) \mid\right] 0, \infty[$ are assumed to be continuously differentiable satisfying

$$
\left.\lim _{e \rightarrow 0} \frac{d u^{i}(x, \cdot)}{d y}\right|_{y=e}=\infty,\left.\lim _{e \rightarrow \infty} \frac{d u^{i}(x, \cdot)}{d y}\right|_{y=e}=0(i=1, \ldots, m)
$$

for $x \geq 0$.

The Inada conditions together with condition (5) imply that for any $i \in\{1, \ldots, m\}$ and every $x \geq 0$ the mapping $\left.\frac{d u^{i}(x, \cdot)}{d y} \mid\right] 0, \infty[$ is injective onto $] 0, \infty[$ with continuously differentiable, strictly decreasing inverse say $I_{i}(x, \cdot)$. Furthermore, in accordance with the investigations of consumption optimization within the setting of expected utility maximizing financial investors the following condition of regularity should be fulfilled:

$$
\mathbb{E}\left[I_{1}\left\{\left(S_{T}, y \mathcal{K}_{\pi}\left(S_{T}\right)\right\}\right], \ldots, \mathbb{E}\left[I_{m}\left\{\left(S_{T}, y \mathcal{K}_{\pi}\left(S_{T}\right)\right\}\right]<\infty \quad \text { for any } y>0\right.\right.
$$

See Dana and Jeanblanc (2003), Duffie (1996), Karatzas and Shreve (1998) for more details.

Theorem 3.1 In addition to (4) - (11) let $\left.u^{1}(x, \cdot) \mid\right] 0, \infty\left[, \ldots, u^{m}(x, \cdot) \mid\right] 0, \infty[$ be twice continuously differentiable for $x \geq 0$.

Then $\left.u_{\alpha}\left(s_{T}, \cdot\right) \mid\right] 0, \infty\left[\right.$ is continuously differentiable for every realization $s_{T}$ of $S_{T}$. Furthermore for any $\alpha_{i}>0$ there exists some $\beta_{i}>0$ such that

$$
\left.\frac{d u_{\alpha}\left(s_{T}, \cdot\right)}{d y}\right|_{y=\bar{e}\left(s_{T}\right)}=\left.\alpha_{i} \frac{d u^{i}\left(s_{T}, \cdot\right)}{d y}\right|_{y=\bar{c}_{i}\left(s_{T}\right)}=\alpha_{i} \beta_{i} \mathcal{K}_{\pi}\left(s_{T}\right)
$$

for every realization $s_{T}$. 
The proof of Theorem 3.1 is delegated to the end of Appendix A.

\section{Remark:}

The relationship between pricing kernels and the marginal utilities of the individual investors as stated in Theorem 3.1 occurs as a motif in the literature of financial mathematics to characterize solutions of different optimization problems. Concerning the optimal utility based investment Kramkov and Schachermayer (1999) introduced a new condition on the asymptotic elasticity of the utilities which replaced the analogue of condition (11). Within our framework it reads as follows

$$
\left.\limsup _{y \rightarrow \infty} \frac{d u^{i}(x, \cdot)}{d y}\right|_{y}<1 \quad \text { for any } x \geq 0 \text { and every } i \in\{1, \ldots, m\} .
$$

Kramkov and Schachermayer restrict themselves to individual investors with classical state independent expected utility preferences. They achieved to show that the new introduced condition is a minimal requirement to describe the optimal investment in terms of the marginal utilities and a pricing kernel. Their methods had been adapted by Karatzas and Zitkovic (2003) to characterize the optimal consumption in incomplete financial market similar to Theorem 3.1. The difference, and the mathematically more challeging point is, that in Karatzas and Zitkovic (2003) the martingale measure is not fixed in advance. Within our setting the guidelines of Kramkov and Schachermayer (1999) might be followed more directly to establish Theorem 3.1 with condition (12) instead of (11). However, a rigorous derivation would lie beyond the scope of this paper, and we use condition (12) which simplifies the argumentation in the proof of Theorem 3.1.

Let $R_{T}=\frac{S_{T}}{S_{0}}$ be the return at maturity. It has a continuous distribution, say $P_{R_{T}}$. If the market endowment specializes to $\bar{e}\left(S_{T}\right)=S_{T} / S_{0}$, Theorem 3.1 reads as follows.

Corollary 3.2 Let $\bar{e}\left(S_{T}\right)=S_{T} / S_{0}$ and let $\left.u^{1}(x, \cdot) \mid\right] 0, \infty\left[, \ldots, u^{m}(x, \cdot) \mid\right] 0, \infty[$ be twice continuously differentiable for $x \geq 0$. Then under (4) - (11), $\left.u_{\alpha}\left(s_{t}, \cdot\right) \mid\right] 0, \infty[$ is continuously differentiable for every realization $r_{T}$, and for any $\alpha_{i}>0$ there exists some $\beta_{i}>0$ such that

$$
\left.\frac{d u_{\alpha}\left(r_{T}, \cdot\right)}{d y}\right|_{y=r_{T}}=\left.\alpha_{i} \frac{d u^{i}\left(r_{T}, \cdot\right)}{d y}\right|_{y=\bar{c}_{i}\left(s_{T}\right)}=\alpha_{i} \beta_{i} \mathcal{K}_{\pi}\left(S_{0} r_{T}\right) \stackrel{\text { def }}{=} \widetilde{\mathcal{K}}_{\pi}\left(r_{T}\right) .
$$

Corollary 3.2 is the corner stone for our microeconomic explanation of the empirical pricing kernel paradox. The framework of state dependent utilities of the investors allows us to describe a switching behaviour of 
them when facing a threshold for the price of the stock at maturity. In more detail, let us assume that each consumer $i$ is disposed of two basic continuous, strictly increasing and strictly concave utility indices $u_{i}^{0}, u_{i}^{1}:\left[0, \infty\left[\rightarrow \mathbb{R} \cup\{-\infty\}\right.\right.$ with $u_{i}^{0}(y), u_{i}^{1}(y) \in \mathbb{R}$ for $y>0$. He or she is changing between these indices dependent on a threshold $x_{i}>0$ for the return $R_{T}$, i.e.

$$
u^{i}\left(r_{T}, \cdot\right)=1_{\left[0, x_{i}\right]}\left(r_{T}\right) u_{i}^{0}+1_{] x_{i}, \infty[}\left(r_{T}\right) u_{i}^{1}
$$

for every realization $r_{T}$ of $R_{T}$. Here $1_{A}$ denotes the indicator function of subset $A$. The reader might think of $u_{i}^{0}, u_{i}^{1}$ as utility indices representing bearish and bullish risk attitudes of consumer $i$, and that her or his revealed attitudes are adapted to the prices of the financial market.

In order to simplify notations, let us assume that the thresholds are ordered by $x_{1} \leq \ldots \leq x_{m}$.

There exist different competing potential representative agent groups in the market with respective representations $U_{\alpha}^{1}\left\{\bar{e}\left(S_{T}\right)\right), \ldots, U_{\alpha}^{m+1}\left(\bar{e}\left(S_{T}\right)\right\}$ of indirect utilities defined by

$$
\begin{aligned}
& U_{\alpha}^{i}\left\{\bar{e}\left(S_{T}\right)\right\} \\
= & \sup \left\{\sum_{k=1}^{i-1} \mathbb{E}\left[e^{-\int_{0}^{R} r_{x} d x} u_{k}^{0}\left\{c_{k}\left(S_{T}\right)\right\}\right]+\sum_{k=i}^{m+1} \mathbb{E}\left[e^{-\int_{0}^{R} r_{x} d x} u_{k}^{1}\left\{c_{k}\left(S_{T}\right)\right\}\right] \mid \sum_{k=1}^{m+1} c_{k}\left(S_{T}\right) \leq \bar{e}\left(S_{T}\right)\right\} .
\end{aligned}
$$

In view of Lemmata B.1, B.2 (cf. Appendix B) they have expected utility representations

$$
U_{\alpha}^{i}\left\{\bar{e}\left(S_{T}\right)\right\}=\mathbb{E}\left[e^{-\int_{0}^{T} r_{x} d x} u_{\alpha}^{i}\left\{\bar{e}\left(s_{T}\right)\right\}\right],
$$

where

$$
u_{\alpha}^{i}(y)=\sup \left\{e^{-\int_{0}^{R} r_{x} d x}\left(\sum_{k=1}^{i-1} \alpha_{k} u_{k}^{0}\left(y_{k}\right)+\sum_{k=i}^{m} \alpha_{k} u_{k}^{1}\left(y_{k}\right)\right) \mid y_{1}, \ldots, y_{k} \geq 0, \sum_{k=1}^{m} y_{k} \leq y\right\}
$$

for $y \geq 0, i \in\{1, \ldots, m+1\}$. It is now a routine excercise to verify that

$$
u_{\alpha}(x, y)=\mathbf{1}_{\left[0, x_{1}\right]}(x) u_{\alpha}^{1}(y)+\sum_{i=1}^{m-1} \mathbf{1}_{] x_{i}, x_{i+1}\right]}(x) u_{\alpha}^{i+1}(y)+1_{] x_{m}, \infty[}(x) u_{\alpha}^{m+1}(y) \text { for } x, y \geq 0 .
$$

As a consequence the indirect utility $U_{\alpha}\left\{\bar{e}\left(S_{T}\right)\right\}$ might be interpreted as expressing the hegemony of the different potential representative agents. Moreover, under the assumptions of Corollary 3.2 we obtain immediately some $\beta>0$ such that

$\left.\mathbf{1}_{\left[0, x_{1}\right]}\left(r_{T}\right) \frac{d u_{\alpha}^{1}\left(r_{T}, \cdot\right)}{d y}\right|_{y=r_{T}}+\left.\sum_{i=1}^{m-1} \mathbf{1}_{\left.] x_{i}, x_{i+1}\right]}\left(r_{T}\right) \frac{d u_{\alpha}^{i+1}\left(r_{T}, \cdot\right)}{d y}\right|_{y=r_{T}}+\left.\mathbf{1}_{] x_{m}, \infty[}\left(r_{T}\right) \frac{d u_{\alpha}^{m+1}\left(r_{T}, \cdot\right)}{d y}\right|_{y=r_{T}}=\beta \widetilde{\mathcal{K}}_{\pi}\left(r_{T}\right)$ 


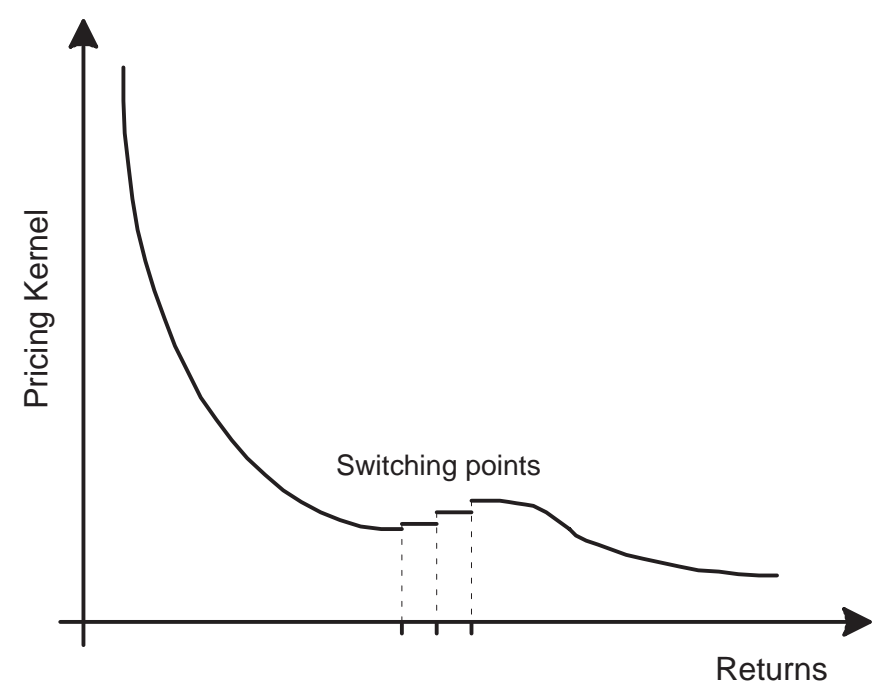

Figure 2: EPK with the switching points.

holds for any realization $r_{T}$ of $R_{T}$. From this observation it becomes clear that the pricing kernel is nonincreasing separately on the intervals $\left[0, x_{1}[,] x_{1}, x_{2}[, \ldots,] x_{m} \infty[\right.$, but it might fail to be monotone just at the switching points $x_{1}, \ldots, x_{m}$ (see figure 2 ).

For illustration let us assume that the distribution of $R_{T}$ has $[0, \infty[$ as support, and that the investors have an identical switching point say $x_{0}$. That means

$$
\left.1_{\left[0, x_{0}\right]}\left(r_{T}\right) \frac{d u_{\alpha}^{1}\left(r_{T}, \cdot\right)}{d y}\right|_{y=r_{T}}+\left.1_{x_{0}, \infty[}\left(r_{T}\right) \frac{d u_{\alpha}^{m+1}\left(r_{T}, \cdot\right)}{d y}\right|_{y=r_{T}}=z \mathcal{K}_{\pi}\left(r_{T}\right)
$$

for every realization $r_{T}$ of $R_{T}$.

Furthermore, let us suppose that each investor $i$ switches between CRRA utilities $u_{i}^{j}(y)=y^{\gamma_{i}^{j}} / \gamma_{i}^{j}(j=0,1)$ with $0<\gamma_{i}^{1}<\gamma_{i}^{0}<1$, inducing the Arrow-Pratt coefficients of absolute risk aversion $\beta_{i}^{j}(y)=\frac{1-\gamma_{i}^{j}}{y}$ $(j=0,1 ; y>0)$. It follows $\beta_{i}^{1}(y)>\beta_{1}^{0}(y)$ for $i \in\{1, \ldots, m\}$ and $y>0$, which means that $u_{1}^{1}, \ldots, u_{m}^{1}$ represent a more risk averse attitude than $u_{1}^{0}, \ldots, u_{m}^{0}$ (cf. Mas-Colell et al. (1995), p. 191). In particular for stock prices lower or equal $x_{0}$ we have a bullish market, whereas we obtain a bearish market when stock prices exceed $x_{0}$.

The application of Lemma B.1 and Proposition B.3 in Appendix B yields

$$
r_{T}=F^{0}\left(\left.\frac{d u_{\alpha}^{1}\left(r_{T}, \cdot\right)}{d y}\right|_{y=r_{T}}\right)=F^{1}\left(\left.\frac{d u_{\alpha}^{m}\left(r_{T}, \cdot\right)}{d y}\right|_{y=r_{T}}\right)
$$


for any positive realization $r_{T}$, where

$$
\left.F^{j}:\right] 0, \infty[\rightarrow] 0, \infty\left[, z \mapsto \sum_{\substack{i=1 \\ \alpha_{i}>0}}^{m} \frac{(z)^{\frac{1}{\gamma_{i}^{j}-1}}}{\alpha_{i}} \quad(j=0,1)\right.
$$

are decreasing bijective mappings. If $x_{0}>\max \left\{F^{0}(1), F^{1}(1)\right\}$, then

$$
\left.\frac{d u_{\alpha}^{1}\left(r_{T}, \cdot\right)}{d y}\right|_{y=r_{T}},\left.\frac{d u_{\alpha}^{m}\left(r_{T}, \cdot\right)}{d y}\right|_{y=r_{T}}<1,
$$

and

$$
F^{0}\left(\left.\frac{d u_{\alpha}^{1}\left(r_{T}, \cdot\right)}{d y}\right|_{y=r_{T}}\right)=r_{T}=F^{1}\left(\left.\frac{d u_{\alpha}^{m}\left(r_{T}, \cdot\right)}{d y}\right|_{y=r_{T}}\right)>F^{0}\left(\left.\frac{d u_{\alpha}^{m}\left(r_{T}, \cdot\right)}{d y}\right|_{y=r_{T}}\right)
$$

for any realization $r_{T} \geq x_{0}$. Therefore

$$
\left.\frac{d u_{\alpha}^{m+1}\left(r_{T}, \cdot\right)}{d y}\right|_{y=r_{T}}>\left.\frac{d u_{\alpha}^{1}\left(r_{T}, \cdot\right)}{d y}\right|_{y=r_{T}} \text { for } r_{T} \geq x_{0}
$$

That means that $\widetilde{\mathcal{K}}_{\pi}$ is not monotone at $x_{0}$.

\section{The Inverse Problems for the Switching Points}

In section 3 we developed an economic environment, where in equilibrium the pricing kernel $\widetilde{\mathcal{K}}_{\pi}\left(R_{T}\right)$ may be expressed by a mixture of different marginal utilities. That means

$$
\widetilde{\mathcal{K}}_{\pi}\left(R_{T}\right)=\left.\sum_{i=1}^{m} \mathbf{1}_{] x_{i-1}, x_{i}\right]}\left(R_{T}\right) \frac{d u_{i}}{d y}\right|_{y=R_{T}}+\left.\mathbf{1}_{] x_{m}, \infty[}\left(R_{T}\right) \frac{d u_{m+1}}{d y}\right|_{y=R_{T}}
$$

for some $m \in \mathbb{N}, 0=x_{0}<\ldots<x_{m}<\infty$ and mappings $u_{1}, \ldots, u_{m+1}:[0, \infty] \rightarrow \mathbb{R} \cup\{-\infty\}$ whose restrictions to $] 0, \infty[$ are strictly increasing, strictly convex twice continuously differentiable real-valued functions satisfying the Inada conditions. Such a representation will be the starting point for the following investigations. The values $x_{1}, \ldots, x_{m}$ will be called the switching points of the pricing kernel $\widetilde{\mathcal{K}}_{\pi}\left(R_{T}\right)$. They are the amounts of returns where the investors are feeling themselves compelled to change their risk attitudes. Usually as a local measure of the risk aversion one might use the Arrow-Pratt coefficient of absolute risk aversion. Hence the change of the risk attitudes might be expressed by drawing a comparison of the left- and right-sided versions the Arrow-Pratt coefficients of absolute risk aversions at the switching 
points. They read as follows

$$
\lim _{\delta \rightarrow 0_{+}} \frac{\frac{\widetilde{\mathcal{K}}_{\pi}\left(x_{i}-\delta\right)-\widetilde{\mathcal{K}}_{\pi}\left(x_{i}\right)}{\delta}}{\widetilde{\mathcal{K}}_{\pi}\left(x_{i}\right)}, \lim _{\delta \rightarrow 0_{+}} \frac{\frac{\widetilde{\mathcal{K}}_{\pi}\left(x_{i}+\delta\right)-\widetilde{\mathcal{K}}_{\pi}\left(x_{i}\right)}{\delta}}{\widetilde{\mathcal{K}}_{\pi}\left(x_{i}\right)} \text { for } i \in\{1, \ldots, m\} .
$$

Unfortunately, neither the number and the location of the switching points nor the marginal utilities of the $u_{1}, \ldots, u_{m+1}$ are known. In order to get an idea of them we suggest to fit basic values of the pricing kernel. For that purpose we fix a set $\mathcal{V}$ of strictly decreasing continuously differentiable mappings $v:] 0, \infty[\rightarrow \mathbb{R}$ satisfying $\lim _{x \rightarrow \infty} v(x)=0$. As a prominent example we mention the parameterized set of functions $v$, defined by $v(x) \stackrel{\text { def }}{=}(x+a)^{b-1}$ for some $a \geq 0$ and $\left.b \in\right] 0,1[$. These are the marginals of the generalized HARA (CRRA) utilities. As test functions for the data fitting we shall use mixtures $\sum_{i=1}^{N} v_{i} \mathbf{1}_{\left.]_{i-1}, x_{i}\right]}$ of functions from $\mathcal{V}$. Here the basic points represent approximately the unknown switching points. The quality of the approximation will be expressed by quadratic mean error w.r.t. a fixed continuous distribution $\hat{F}$ with density function say $\hat{p}$ and compact support enclosed in $[0, \infty[$. The mapping $\hat{p}$ might be a kernel estimation of a density function for the distribution of $R_{T}$. Henceforth we shall denote the minimum and maximum of the support by $\underline{z}$ and $\bar{z}$ respectively.

We shall focus on the choice of appropriate basic points. The two approaches we want to propose differ in the way to discretize the pricing kernel.

\subsection{The Monte Carlo Approach}

For any distribution $F$ on $\mathbb{R}$ satisfying $F\left(\left[\underline{z}, \infty[)=1\right.\right.$, we shall consider i.i.d. sequences $\left(X_{N}^{F}\right)_{N}$ of random variables with common distribution $F$. Fixing $N$, they are associated with the order statistics $X_{1: N}, \ldots, X_{N: N}$ according to $\underline{z} \stackrel{\text { def }}{=} X_{0: N} \leq X_{1: N} \leq \ldots \leq X_{N: N}$.

The idea is to discretize randomly $\widetilde{\mathcal{K}}_{\pi}$ by $\widetilde{\mathcal{K}}_{\pi}^{N}(F) \stackrel{\text { def }}{=} \sum_{i=1}^{N} \widetilde{\mathcal{K}}_{\pi}\left(X_{i: N}^{F}\right) \mathbf{1}_{\left.] X_{(i-1): N}^{F}, X_{i: N}^{F}\right]}$. The suggestion is statistically motivated by the following observation.

Proposition 4.1 Let $F$ be a continuous distribution on $\mathbb{R}$ with $F\left(\left[\underline{z}, \infty[)=1\right.\right.$, and let $\mathcal{C}\left(\widetilde{\mathcal{K}}_{\pi}\right)$ denote the set of continuity points of $\widetilde{\mathcal{K}}_{\pi}$. Then for any $x \in \mathcal{C}\left(\widetilde{\mathcal{K}}_{\pi}\right)$ which belongs to the topological interior int $(\operatorname{supp}(F))$ of the support of $F$ we obtain

$$
\sum_{i=1}^{N} \widetilde{\mathcal{K}}_{\pi}\left(X_{i: N}^{F}\right) \mathbf{1}_{] X_{(i-1): N}^{F}, X_{i: N}^{F}\right]}(x) \rightarrow \widetilde{\mathcal{K}}_{\pi}(x) \text { a.s.. }
$$


In particular, setting $i(F) \stackrel{\text { def }}{=} \inf \{x \mid F(]-\infty, x])>0\}$ and $s(F) \stackrel{\text { def }}{=} \sup \{x \mid F(]-\infty, x])<1\}$, we may even achieve this convergence for any $x \in \mathcal{C}\left(\widetilde{\mathcal{K}}_{\pi}\right) \cap i(F), s(F)[$ if supp $(F)$ is konvex.

Proposition 4.1 is an immediate application of Proposition C.1 in appendix C.

The choice of proper basic points for the discretization changes into the problem to find a proper a priori distribution for the Monte Carlo sampling. The sample size $N$ will be assumed to be given exogeneously. Simulating the jumping behaviour of $\widetilde{\mathcal{K}}_{\pi}$ in finite many points, we shall restrict ourselves to all continuous distributions with bounded support enclosed in $[0, \infty[$, and which have density functions being constant on each interval $] x_{i-1}, x_{i}\left[(i=1, \ldots, N)\right.$ for some $x_{0} \leq x_{1} \leq \ldots \leq x_{N}$, where $x_{0}$ and $x_{N}$ denote a lower and an upper bound of the support respectively. In particular we consider the set $\mathcal{P}_{N}$ of all continuous distributions with bounded support enclosed in $[\underline{z}, \bar{z}]$, and which have density functions being constant on each interval $] x_{i-1}, x_{i}\left[(i=1, \ldots, N)\right.$ for some $\underline{z} \stackrel{\text { def }}{=} x_{0} \leq x_{1} \leq \ldots \leq x_{N} \stackrel{\text { def }}{=} \bar{z}$. Furthermore for $F \in \mathcal{P}_{N}$ we shall denote the joint distribution of $X_{1: N}^{F}, \ldots, X_{N: N}^{F}$ by $F_{(N)}$. Then the inverse problem reads as follows.

\section{Inverse problem:}

Find $F^{*} \in \mathcal{P}_{N}$ such that

$$
\begin{aligned}
& \inf _{v_{1}, \ldots, v_{N} \in \mathcal{V}} \int \mathbb{E}_{F_{(N)}^{*}}\left[\widetilde{\mathcal{K}}_{\pi}^{N}\left(F^{*}\right)(x)-\sum_{i=1}^{N} v_{i}(x) \mathbf{1}_{] X_{(i-1): N}^{F^{*}}, X_{i: N}^{\left.F^{*}\right]}\right]}(x)\right]^{2} \hat{p}(x) d x \\
= & \min _{F \in \mathcal{P}_{N}(z) v_{1}, \ldots, v_{N} \in \mathcal{V}} \inf _{\mathbb{E}} \int \mathbb{E}_{F_{(N)}}\left[\widetilde{\mathcal{K}}_{\pi}^{N}(F)(x)-\sum_{i=1}^{N} v_{i}(x) \mathbf{1}_{] X_{(i-1): N}^{F}, X_{i: N}^{F}\right]}(x)\right]^{2} \hat{p}(x) d x
\end{aligned}
$$

We shall discuss the solvability of the inverse problem after we shall have introduced the second inverse problem to be considered.

\subsection{The pure numerical approach}

The restriction $\widetilde{\mathcal{K}}_{\pi} \mid[\underline{z}, \bar{z}]$ should be discretized by by some basic values. More precisely, we choose a partition $\underline{z} \stackrel{\text { def }}{=} x_{0} \leq x_{1}<\ldots<x_{N} \stackrel{\text { def }}{=} \bar{z}$ of $[\underline{z}, \bar{z}]$, and use $\widetilde{\mathcal{K}}_{\pi}^{N}\left(x_{1}, \ldots, x_{N}\right) \stackrel{\text { def }}{=} \sum_{i=1}^{N} \widetilde{\mathcal{K}}_{\pi}\left(x_{i}\right) \mathbf{1}_{\left.] x_{i-1}, x_{i}\right]}$ as an approximation of $\widetilde{\mathcal{K}}_{\pi} \mid[\underline{z}, \bar{z}]$. This idea suggests itself by the following observation.

Proposition 4.2 The mapping $\sum_{i=1}^{N} \widetilde{\mathcal{K}}_{\pi}\left(a+\frac{i}{N}(b-a)\right) \mathbf{1}_{\left.] a+\frac{(i-1)}{N}(b-a), a+\frac{i}{N}(b-a)\right]}$ converges to $\widetilde{\mathcal{K}}_{\pi} \mid[a, b]$ uniformly on compacta of continuity points of $\widetilde{\mathcal{K}}_{\pi} \mid[a, b]$ for any nondegenerated interval $\left.[a, b] \subseteq\right] 0, \infty[$. 
Proposition 4.2 may be derived immediately from the well known Korovkin like approximation result for mappings on $[0,1]$ (cf. e.g. Witting and Müller-Funk (1995), Satz B5.2).

As for the Monte Carlo approach we shall not care about the choice of $N$, it will be assumed to be exogeneously given. Denoting by $\mathcal{Z}_{N}$ the set of all $\left(x_{1}, \ldots, x_{N}\right) \in \mathbb{R}^{N}$ satisfying $x_{0} \stackrel{\text { def }}{=} \underline{z} \leq x_{1} \leq \ldots \leq x_{N}=\bar{z}$, the choice of the basic points might be described in terms of the following inverse problem.

\section{Inverse problem:}

Find $\left(x_{1}^{*}, \ldots, x_{N}^{*}\right) \in \mathcal{Z}_{N}$ such that

$$
\begin{aligned}
& \inf _{v_{1}, \ldots, v_{N} \in \mathcal{V}} \int \sum_{i=1}^{N}\left[\widetilde{\mathcal{K}}_{\pi}\left(x_{i}^{*}\right)-v_{i}(x)\right]^{2} \mathbf{1}_{] x_{(i-1)}^{*}, x_{i}^{*}\right]}(x) \hat{p}(x) d x \\
= & \inf _{\left(x_{0}, \ldots, x_{N}\right) \in \mathcal{Z}_{N}} \inf _{v_{1}, \ldots, v_{N} \in \mathcal{V}} \int \sum_{i=1}^{N}\left[\widetilde{\mathcal{K}}_{\pi}\left(x_{i}\right)-v_{i}(x)\right]^{2} \mathbf{1}_{\left.x_{(i-1)}, x_{i}\right]}(x) \hat{p}(x) d x
\end{aligned}
$$

As a convention we tacitly set $\widetilde{\mathcal{K}}_{\pi}(0) \stackrel{\text { def }}{=} \infty$ and $\infty \cdot 0 \stackrel{\text { def }}{=} 0$.

\subsection{Solvability of the inverse problems}

Throughout this section we shall assume

$$
\sup _{v \in \mathcal{V}} \int v(x)^{2+\delta} \hat{p}(x) d x<\infty \text { for some } \delta>0
$$

Remark 4.3 In the following situations the family $\mathcal{V}$ fulfills the assumed integrability condition:

$$
\begin{aligned}
& \text { 1. } \int_{0}^{1} \frac{\hat{p}(x)}{x^{2+\delta}} d x<\infty \text { for some } \delta>0 \text {, and } \mathcal{V} \stackrel{\text { def }}{=}\{v:] 0, \infty\left[\rightarrow \mathbb{R}, x \mapsto(x+a)^{b-1} \mid a \geq 0, b \in\right] 0,1[\} \text {. } \\
& \text { 2. } \underline{z}>0 \text {, and } \sup _{v \in \mathcal{V}} v(\underline{z})<\infty \text {, e.g. if } \mathcal{V} \stackrel{\text { def }}{=}\{v:] 0, \infty\left[\rightarrow \mathbb{R}, x \mapsto(x+a)^{b-1} \mid a \geq 0, b \in\right] 0,1[\} \text {. }
\end{aligned}
$$

The integrability condition means that $\mathcal{V}$ is $L_{2}-$ norm bounded. In particular $\mathcal{V}$ as well as $\left\{v^{2} \mid v \in \mathcal{V}\right\}$ are uniformly $\hat{F}$-integrable, and the weak closure $c l^{w}(\mathcal{V})$ of $\mathcal{V}$ is weakly compact because $L_{2}(\hat{F})$, equipped with the $L_{2}-$ norm, is a reflexive Banach space. Moreover, since the standard Borel $\sigma$-algebra on $\mathbb{R}$ is countably generated, the $L_{2}-$ norm topology on $L_{2}(\hat{F})$ is separable. Hence the relative topology of the weak topology to $c l^{w}(\mathcal{V})$ is metrizable (cf. Dunford and Schwarz (1958), Theorem V.6.3), and thus as a compact topology also separable. So we obtain the following result. 
Lemma 4.4 Under the integrability condition on $\mathcal{V}$ the weak closure $c l^{w}(\mathcal{V})$ of $\mathcal{V}$ is weakly compact, and the relative weak topology to $\mathrm{cl}^{w}(\mathcal{V})$ is separably metrizable.

For abbreviation let us define $g: \mathcal{Z}_{N} \times L_{2}(\hat{F})^{N} \rightarrow \mathbb{R} \cup\{\infty\}$ by

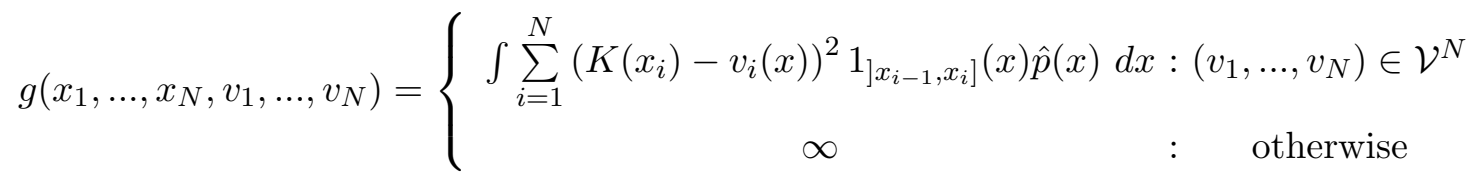

Let us gather some basic properties of $g$.

Lemma 4.5 Let $\tau_{1}$ be the relative topology of the standard topology on $\mathbb{R}^{N}$ to $\mathcal{Z}_{N}$, let $\tau_{2}$ denote the product weak topology on $L_{2}(\hat{F})^{N}$, and let $\tau_{1} \times \tau_{2}$ stand for the product topology of $\tau_{1}$ and $\tau_{2}$. Furthermore let us denote the set of continuity points of $\widetilde{\mathcal{K}}_{\pi}$ by $\mathcal{C}\left(\widetilde{\mathcal{K}}_{\pi}\right)$. Then under the integrability condition on $\mathcal{V}$ the mapping $g$ satisfies the following properties:

1. $g\left(x_{1}, \ldots, x_{N}, \cdot\right) \mid \mathcal{V}^{N}$ is lower continuous w.r.t. $\tau_{2}$ for every $\left(x_{1}, \ldots, x_{N}\right) \in \mathcal{Z}_{N}$;

2. $g\left(\cdot, v_{1}, \ldots, v_{N}\right)$ is measurable w.r.t. the Borel $\sigma$-algebra generated by $\tau_{1}$ for any $\left(v_{1}, \ldots, v_{N}\right) \in \mathcal{V}^{N}$, and the restrictions of these mappings to $\mathcal{Z}_{N} \cap \mathcal{C}\left(\widetilde{\mathcal{K}}_{\pi}\right)^{N}$ are even continuous w.r.t. $\tau_{1}$;

3. The family $\left\{g(\cdot, \underline{v})\left|\mathcal{Z}_{N} \cap \mathcal{C}\left(\widetilde{\mathcal{K}}_{\pi}\right)^{N}\right| \underline{v} \in \mathcal{V}^{N}\right\}$ is equicontinuous w.r.t. $\tau_{1}$, in particular the restriction of $g$ to $\left(\mathcal{Z}_{N} \cap \mathcal{C}\left(\widetilde{\mathcal{K}}_{\pi}\right)^{N}\right) \times \mathcal{V}^{N}$ is lower semicontinuous w.r.t. $\tau_{1} \times \tau_{2}$.

\section{Proof:}

The statement 1. may be verified by routine procedures. For that purpose it should be observed that we may restrict ourselves to sequential weak lower semicontinuity by Lemma 4.4 and that the squared $L_{2}-$ norm is weakly lower semicontinuous as a convex $L_{2}-$ norm continuous mapping.

Statement 2. follows easily from measurability of $\widetilde{\mathcal{K}}_{\pi}$ and the following observation

$\left(^{*}\right)$ For every $\varepsilon_{1}>0$, there is some $\varepsilon_{2}>0$ such that

$$
\sup _{w \in \mathcal{W}} \int|w(x)|\left|\mathbf{1}_{] y_{1}, y_{2}\right]}(x)-\mathbf{1}_{] z_{1}, z_{2}\right]}(x)\right| \hat{p}(x) d x<\varepsilon_{1}
$$

whenever $\left(y_{1}, y_{2}\right),\left(z_{1}, z_{2}\right) \in\left[0, \infty\left[^{2}\right.\right.$ with $y_{1} \leq y_{2}, z_{1} \leq z_{2}$ and $\left(y_{1}-z_{1}\right)^{2}+\left(y_{2}-z_{2}\right)^{2}<\varepsilon_{2}$. Here $\mathcal{W}$ denotes an arbitrary familiy of uniformly $\hat{F}$-integrable real-valued mappings on $] 0, \infty[$. 
In order to see $\left(^{*}\right)$ observe $\int\left|\mathbf{1}_{\left.] y_{1}, y_{2}\right]}-\mathbf{1}_{\left.] z_{1}, z_{2}\right]}\right| \hat{p}(x) d x=\int\left|\mathbf{1}_{] y_{1}, y_{2}[}-\mathbf{1}_{] z_{1}, z_{2}}\right| \hat{p}(x) d x \rightarrow 0$ as $\left(z_{1}, z_{2}\right) \rightarrow$ $\left(y_{1}, y_{2}\right)$, and that $\left|\mathbf{1}_{\left.] y_{1}, y_{2}\right]}-\mathbf{1}_{\left.] z_{1}, z_{2}\right]}\right|$ is just indicator function of the symmetric difference of the involved intervals.

For the proof of statement 3 . it suffices to show $\tau_{1}$-equicontinuity of $\left\{g(\cdot, \underline{v})\left|\mathcal{Z}_{N} \cap \mathcal{C}\left(\widetilde{\mathcal{K}}_{\pi}\right)^{N}\right| \underline{v} \in \mathcal{V}^{N}\right\}$ because the second part of statement 3 . follows then in view of statement 1 .. So let us fix some $\left(x_{1}, \ldots, x_{n}\right) \in$ $\mathcal{Z}_{N} \cap \mathcal{C}\left(\widetilde{\mathcal{K}}_{\pi}\right)^{N}$. Since $\mathcal{Z}_{N} \cap \mathcal{C}\left(\widetilde{\mathcal{K}}_{\pi}\right)^{N} \in \tau_{1}$, we may find a bounded neighbourhood $U \in \tau_{1}$ of $\left(x_{1}, \ldots, x_{n}\right)$ such that $U \subseteq \mathcal{C}\left(\widetilde{\mathcal{K}}_{\pi}\right)^{N} \cap[\rho, \bar{z}]^{N}$ for some $\rho>0$. Then we obtain for any $\left(\tilde{x}_{1}, \ldots, \tilde{x}_{N}\right) \in U$ and any $\left(v_{1}, \ldots, v_{N}\right) \in \mathcal{V}^{N}$

$$
\begin{aligned}
& \left|g\left(x_{1}, \ldots, x_{N}, v_{1}, \ldots, v_{N}\right)-g\left(\tilde{x}_{1}, \ldots, \tilde{x}_{N}, v_{1}, \ldots, v_{N}\right)\right| \\
\leq \quad & \sum_{i=1}^{N} \int\left(\widetilde{\mathcal{K}}_{\pi}\left(x_{i}\right)-v_{i}(x)\right)^{2}\left|\mathbf{1}_{] x_{i-1}, x_{i}\right]}-\mathbf{1}_{] \tilde{x}_{i-1}, \tilde{x}_{i}\right]}\right| \hat{p}(x) d x \\
+ & \sum_{i=1}^{N} \int\left|\left(\widetilde{\mathcal{K}}_{\pi}\left(x_{i}\right)-v_{i}(x)\right)^{2}-\left(\widetilde{\mathcal{K}}_{\pi}\left(\tilde{x}_{i}\right)-v_{i}(x)\right)^{2}\right| \mathbf{1}_{] \tilde{x}_{i-1}, \tilde{x}_{i}\right]} \hat{p}(x) d x \\
\leq \quad & \sum_{i=1}^{N} \sup _{v \in \mathcal{V}} \int\left(\widetilde{\mathcal{K}}_{\pi}\left(x_{i}\right)-v(x)\right)^{2}\left|\mathbf{1}_{] x_{i-1}, x_{i}\right]}-\mathbf{1}_{] \tilde{x}_{i-1}, \tilde{x}_{i}\right]}\right| \hat{p}(x) d x \\
+ & \sum_{i=1}^{N} \sup _{v \in \mathcal{V}} \int\left|\left(\widetilde{\mathcal{K}}_{\pi}\left(x_{i}\right)-v(x)\right)^{2}-\left(\widetilde{\mathcal{K}}_{\pi}\left(\tilde{x}_{i}\right)-v(x)\right)^{2}\right| \mathbf{1}_{] \tilde{x}_{i-1}, \tilde{x}_{i}\right]} \hat{p}(x) d x \\
\leq \quad & \sum_{i=1}^{N} \sup _{v \in \mathcal{V}} \int\left(\widetilde{\mathcal{K}}_{\pi}\left(x_{i}\right)-v(x)\right)^{2}\left|\mathbf{1}_{] x_{i-1}, x_{i}\right]}-\mathbf{1}_{] \tilde{x}_{i-1}, \tilde{x}_{i}\right]}\right| \hat{p}(x) d x \\
+ & \sum_{i=1}^{N}\left|\widetilde{\mathcal{K}}_{\pi}\left(x_{i}\right)-\widetilde{\mathcal{K}}_{\pi}\left(\tilde{x}_{i}\right)\right| \sup _{v \in \mathcal{V}} \int\left(\widetilde{\mathcal{K}}_{\pi}\left(x_{i}\right)+\widetilde{\mathcal{K}}_{\pi}\left(\tilde{x}_{i}\right)+2 v(x)\right) \hat{p}(x) d x
\end{aligned}
$$

Since we have assumed $\widetilde{\mathcal{K}}_{\pi}$ to be a piecewise decreasing positive function, $\widetilde{\mathcal{K}}_{\pi} \mid[\rho, \bar{z}]$ is bounded from above by some positive $\delta$. Additionally, $\sup _{v \in \mathcal{V}} \int v(x) \hat{p}(x) d x<\infty$ by weak compactness of $c l^{w}(\mathcal{V})$, and $\left\{v^{2} \mid v \in \mathcal{V}\right\}$ is uniformly $\hat{F}$-integrable due the integrability condition on $\mathcal{V}$. Therefore, on one hand

$$
\begin{aligned}
& \sum_{i=1}^{N}\left|\widetilde{\mathcal{K}}_{\pi}\left(x_{i}\right)-\widetilde{\mathcal{K}}_{\pi}\left(\tilde{x}_{i}\right)\right| \sup _{v \in \mathcal{V}} \int\left(\widetilde{\mathcal{K}}_{\pi}\left(x_{i}\right)+\widetilde{\mathcal{K}}_{\pi}\left(\tilde{x}_{i}\right)+2 v(x)\right) \hat{p}(x) d x \\
\leq & \left(2 \delta+2 \sup _{v \in \mathcal{V}} \int v(x) \hat{p}(x) d x\right) \sum_{i=1}^{N}\left|\widetilde{\mathcal{K}}_{\pi}\left(x_{i}\right)-\widetilde{\mathcal{K}}_{\pi}\left(\tilde{x}_{i}\right)\right| .
\end{aligned}
$$


On the other hand $\left\{[K(x)-v]^{2} \mid x \in[\rho, \bar{z}], v \in \mathcal{V}\right\}$ is uniformly $\hat{F}$-integrable (cf. Bauer (1992), Korollar 21.3), and we may apply $\left(^{*}\right)$. Putting all together, the equicontinuity of $\left\{g(\cdot, \underline{v})\left|\mathcal{Z}_{N} \cap \mathcal{C}\left(\widetilde{\mathcal{K}}_{\pi}\right)^{N}\right| \underline{v} \in \mathcal{V}^{N}\right\}$ at $\left(x_{1}, \ldots, x_{N}\right)$ follows immediately, completing proof.

Next let us introduce the lower semicontinuous envelope $l s c(g)$ of $g$, defined to be the largest $\tau_{1} \times \tau_{2}-$ lower semicontinuous $\mathbb{R} \cup\{\infty\}$-valued mapping dominated by $g$. Notice that $g$ and $l s c(g)$ coincide on the set $\left(\mathcal{Z}_{N} \cap \mathcal{C}\left(\widetilde{\mathcal{K}}_{\pi}\right)^{N}\right) \times \mathcal{V}^{N}$ by Proposition 3.6 in DalMaso (1993).

The lower semicontinuous envelope will turn out to be a useful tool concerning the solvability of the inverse problems posed in the subsections before. Let us begin with the one coming out of the pure numerical approach. In terms of $g$ it reads as follows.

$$
\begin{aligned}
& \operatorname{minimize} \inf _{\underline{v} \in \mathcal{V}^{N}} g\left(x_{1}, \ldots, x_{N}, \underline{v}\right) \\
& \text { among all }\left(x_{1}, \ldots, x_{N}\right) \in \mathcal{Z}_{N} .
\end{aligned}
$$

Fortunately, we may apply directly Theorem 3.8 in DalMaso (1993), observing that $\mathcal{Z}_{N} \times c l^{w}(\mathcal{V})^{N}$ is compact and sequentially compact w.r.t. $\tau_{1} \times \tau_{2}$, and by Lemma 4.5

$$
\lim _{m \rightarrow \infty}\left|\inf _{\underline{v} \in \mathcal{V}^{N}} g\left(\underline{x}_{m}, \underline{v}\right)-\inf _{\underline{v} \in \mathcal{V}^{N}} g\left(\underline{x}_{0}, \underline{v}\right)\right| \leq \lim _{m \rightarrow \infty} \sup _{\underline{v} \in \mathcal{V}^{N}}\left|g\left(\underline{x}_{m}, \underline{v}\right)-g\left(\underline{x}_{0}, \underline{v}\right)\right|=0
$$

for any sequence $\left(\underline{x}_{m}\right)_{m \in \mathbb{N}_{0}}$ in $\mathcal{Z}_{N} \cap \mathcal{C}\left(\widetilde{\mathcal{K}}_{\pi}\right)^{N} \in \tau_{1}$ with $\underline{x}_{m} \rightarrow \underline{x}_{0}$.

Theorem 4.6 Let $\left(\left(\underline{x}_{m}, \underline{v}_{m}\right)\right)_{m}$ be a sequence in $\mathcal{Z}_{N} \times \mathcal{V}^{N}$ such that $\lim _{m \rightarrow \infty} g\left(\underline{x}_{m}, \underline{v}_{m}\right)=\inf _{\underline{x} \in \mathcal{Z}_{N}} \inf _{\underline{v} \in \mathcal{V}^{N}} g(\underline{x}, \underline{v})$. Then this sequence has at least one cluster point in $\mathcal{Z}_{N} \times c l^{w}(\mathcal{V})^{N}$, and every such cluster point $\left(\underline{x}^{*}, \underline{v}^{*}\right)$ satisfies

$$
l s c(g)\left(\underline{x}^{*}, \underline{v}^{*}\right)=\min _{(\underline{x}, \underline{v}) \in \mathcal{Z}_{N} \times c l^{w}(\mathcal{V})^{N}} l s c(g)\left((\underline{x}, \underline{v})=\inf _{(\underline{x}, \underline{v}) \in \mathcal{Z}_{N} \times \mathcal{V}^{N}} g(\underline{x}, \underline{v}),\right.
$$

with $\inf _{\underline{v} \in \mathcal{V}^{N}} g\left(\underline{x}^{*}\right)=\inf _{\underline{x} \in \mathcal{Z}_{N}} \inf _{\underline{v} \in \mathcal{V}^{N}} g(\underline{x}, \underline{v})$ if the components of $\underline{x}^{*}$ are continuity points of $\widetilde{\mathcal{K}}_{\pi}$.

In view of Tonelli's theorem the inverse problem we have formulated after introducing the Monte-Carlo approach may be described in terms of $g$ by

$$
\begin{aligned}
\operatorname{minimize} & \inf _{\underline{v} \in \mathcal{V}^{N}} \mathbb{E}_{F_{(N)}}\left[g\left(X_{1: N}^{F}, \ldots, X_{N: N}^{F}, \underline{v}\right)\right] \\
& \text { among all } F \in \mathcal{P}_{N} .
\end{aligned}
$$

Concerning the solvability of this problem we may observe the following result. 
Theorem 4.7 Let $\tau_{w}$ be the topology of weak convergence on the set of distributions on $\mathbb{R}$, and let $\tau_{w} \times \tau_{2}$ denote the product topology of $\tau_{w}$ and the product weak topology $\tau_{2}$ on $L_{2}(\hat{F})^{N}$. Furthermore let us consider a sequence $\left(\left(F_{m}, \underline{v}_{m}\right)\right)_{m}$ in $\mathcal{P}_{N} \times \mathcal{V}^{N}$ fulfilling

$$
\lim _{m \rightarrow \infty} \mathbb{E}_{F_{m(N)}}\left[g\left(X_{1: N}^{F_{m}}, \ldots, X_{N: N}^{F_{m}}, \underline{v}_{m}\right)\right]=\inf _{F \in \mathcal{P}_{N}} \inf _{\underline{v} \in \mathcal{V}^{N}} \mathbb{E}_{F_{(N)}}\left[g\left(X_{1: N}^{F}, \ldots, X_{N: N}^{F}, \underline{v}\right)\right] .
$$

Then this sequence has a cluster point w.r.t. to $\tau_{w} \times \tau_{2}$. Every such cluster point $\left(F^{*}, \underline{v}^{*}\right)$ satisfies

$$
\begin{aligned}
& \min \left\{\liminf _{m \rightarrow \infty} \mathbb{E}_{F_{m(N)}}\left[g\left(X_{1: N}^{F_{m}}, \ldots, X_{N: N}^{F_{m}}, \underline{\tilde{v}}_{m}\right)\right] \mid \underline{\tilde{v}}_{m} \rightarrow \underline{v}^{*}\right\} \\
= & \min _{\underline{v} \in L_{2}(\hat{F})^{N}} \min \left\{\liminf _{m \rightarrow \infty} \mathbb{E}_{F_{m(N)}}\left[g\left(X_{1: N}^{F_{m}}, \ldots, X_{N: N}^{F_{m}}, \underline{\tilde{v}}_{m}\right)\right] \mid \underline{\tilde{v}}_{m} \rightarrow \underline{v}\right\} \\
= & \inf _{F \in \mathcal{P}_{N}} \inf _{\underline{v} \in \mathcal{V}^{N}} \mathbb{E}_{F_{(N)}}\left[g\left(X_{1: N}^{F}, \ldots, X_{N: N}^{F}, \underline{v}\right)\right],
\end{aligned}
$$

where even $\inf _{\underline{v} \in \mathcal{V}^{N}} \mathbb{E}_{F^{*}}\left[g\left(X_{1: N}^{F}, \ldots, X_{N: N}^{F}, \underline{v}\right)\right]=\inf _{F \in \mathcal{P}_{N}} \inf _{v \in \mathcal{V}^{N}} \mathbb{E}_{F_{(N)}}\left[g\left(X_{1: N}^{F}, \ldots, X_{N: N}^{F}, \underline{v}\right)\right]$ holds if $\underline{z}>0$, and the set of discontinuity points of $\widetilde{\mathcal{K}}_{\pi}$ is a $F^{*}$-null set (e.g. if $F^{*}$ is a continuous distribution).

\section{Proof:}

First of all, since the topology of weak convergence for distributions on $\mathbb{R}$ is completely as well as separably metrizable, and since the supports of the distributions $F_{m}$ are uniformly enclosed in a compact subset of $\mathbb{R}$, the sequence $\left(F_{m}\right)_{m}$ is uniformly tight, and therefore has a cluster point by Prokhorov's theorem. Furthermore $c l^{w}(\mathcal{V})^{N}$ has been observed as sequentially $\tau_{2}$-compact by Lemma 4.4, so that we may conclude that $\left(\left(F_{m}, \underline{v}_{m}\right)\right)_{m}$ has a cluster point w.r.t. $\tau_{w} \times \tau_{2}$.

Now, let $\left(F^{*}, \underline{v}^{*}\right)$ be a cluster point of $\left(\left(F_{m}, \underline{v}_{m}\right)\right)_{m}$. Without loss of generality we may assume that it is even a limit point. Then the induced sequence $\left(F_{m(N)}\right)_{m}$ of the respective joint distributions of the order statistics $X_{1: N}^{F_{m}}, \ldots, X_{N: N}^{F_{m}}$ has the joint distribution $F_{(N)}^{*}$ of $X_{1: N}^{F^{*}}, \ldots, X_{N: N}^{F^{*}}$ as a limit point w.r.t. the topology of weak convergence on the set of distributions on $\mathbb{R}^{N}$. Indeed, denoting the joint distribution of the order statistics $U_{1: N}, \ldots, U_{N: N}$ obtained from an i.i.d sample of size $N$ according to the uniform distribution on $] 0,1\left[\right.$ by $F_{U,(N)}$, we have $\left.\left.\left.\left.\left.\left.F_{m(N)}\left(\underset{i=1}{\mathbb{X}_{x}}\right]-\infty, x_{i}\right]\right)=F_{U,(N)}\left(\underset{i=1}{X_{X}^{N}}\right]-\infty, F_{m}(]-\infty, x_{i}\right]\right)\right]\right)$ for any $\left(x_{1}, \ldots, x_{N}\right) \in \mathbb{R}^{N}$, and an analogous expression for $F_{N}^{*}$. Then the claim follows immediately from the Helly Bray theorem. 
Now we may apply directly Theorem 7.8 from DalMaso (1993) to the sequence $\left(G_{m}\right)_{m}$ of functions $G_{m}$ : $L_{2}(\hat{F})^{N} \rightarrow \mathbb{R} \cup\{\infty\}$, defined by

$$
G_{m}(\underline{v}) \stackrel{\text { def }}{=}\left\{\begin{array}{cc}
\mathbb{E}_{F_{m(N)}}\left[g\left(X_{1: N}^{F_{m}}, \ldots, X_{N: N}^{F_{m}}, \underline{v}\right)\right] & : \underline{v} \in \mathcal{V}^{N} \\
\infty & : \text { otherwise }
\end{array}\right.
$$

This proves the first part of the Theorem 4.7.

In the case of $\underline{z}>0$, the mapping $\widetilde{\mathcal{K}}_{\pi} \mid[\underline{z}, \bar{z}]$ is bounded, because as a result of section 3 we may describe $\widetilde{\mathcal{K}}_{\pi}$ by $\widetilde{\mathcal{K}}_{\pi}=\sum_{i=1}^{k} \mathbf{1}_{\left.y_{i-1}, y_{i}\right]} w_{i}$, where $0=y_{0}<y_{1}<\ldots<y_{k}=\infty$, and $w_{1}, \ldots, w_{k}$ denote decreasing real-valued mappings on $] 0, \infty[$. This implies that $g(\cdot, \underline{v})$ is bounded too for every $\underline{v} \in \mathcal{V}$.

Moreover, $\left[X_{i: N}^{F}=x\right]$ is a null set for any distribution $F$ whose distribution function is continuous at $x$. Hence, denoting the set of continuity points of $\widetilde{\mathcal{K}}_{\pi}$ by $\mathcal{C}\left(\widetilde{\mathcal{K}}_{\pi}\right)^{N}$, we have $F_{(N)}\left(\mathcal{Z}_{N} \times \mathcal{C}\left(\widetilde{\mathcal{K}}_{\pi}\right)^{N}\right)=1$ for every $F \in\left\{F_{m}, F^{*} \mid m \in \mathbb{N}\right\}$ because by assumption $\widetilde{\mathcal{K}}_{\pi}$ is not continuous at finitely many points only. Therefore in view of Lemma 4.5 we might draw on a well known result concerning uniform convergence w.r.t. the topologies of weak convergence (cf. Billingsley and Topsoe (1967), Theorem 1) to conclude

$$
\begin{aligned}
& \left|\inf _{\underline{v} \in \mathcal{V}^{N}} \mathbb{E}_{F_{m(N)}}\left[g\left(X_{1: N}^{F_{m}}, \ldots, X_{N: N}^{F_{m}}, \underline{v}\right)\right]-\inf _{\underline{v} \in \mathcal{V}^{N}} \mathbb{E}_{F_{(N)}^{*}}\left[g\left(X_{1: N}^{F^{*}}, \ldots, X_{N: N}^{F^{*}}, \underline{v}\right)\right]\right| \\
\leq & \sup _{\underline{v} \in \mathcal{V}^{N}} \mid \mathbb{E}_{F_{m(N)}}\left[g\left(X_{1: N}^{F_{m}}, \ldots, X_{N: N}^{F_{m}}, \underline{v}\right]-\mathbb{E}_{F_{(N)}^{*}}\left[g\left(X_{1: N}^{F^{*}}, \ldots, X_{N: N}^{F^{*}}, \underline{v}\right] \mid \rightarrow 0 .\right.\right.
\end{aligned}
$$

The proof is complete.

\section{A Appendix}

We continue with the consumption model of section 3, retaking all assumptions and notations. The aim of this section is to provide a proof for Theorem 3.1. For this purpose let us firstly characterize the optimal consumptions $\bar{c}_{1}\left(S_{T}\right), \ldots, \bar{c}_{m}\left(S_{T}\right)$ of the individual consumer.

Assumption (3.5) enables us to apply the dominated convergence theorem to show

(A1) continuity of the mappings

$$
\left.g_{s_{t}}^{i}:\right] 0, \infty\left[\rightarrow \mathbb{R}, y \mapsto I_{i}\left\{s_{T}, y \mathcal{K}_{\pi}\left(s_{T}\right)\right\} \mathcal{K}_{\pi}\left(s_{T}\right)\left(s_{T} \geq 0, i \in\{1, \ldots, m\}\right) .\right.
$$


Furthermore the Inada conditions together with monotone and dominated convergence imply

(A2) $\lim _{y \rightarrow 0} g_{s_{T}}^{i}(y)=\infty$ and $\lim _{y \rightarrow \infty} g_{s_{T}}^{i}(y)=0$.

We are now ready to extend the classical characterization of the optimal consumption to the case of extended expected utility preferences.

Theorem A.1 Assuming (4) - (11), there exist $y_{1}, \ldots, y_{m}>0$ such that

$$
\bar{c}_{i}\left(S_{T}\right)=I_{i}\left\{S_{T}, y_{i} \mathcal{K}_{\pi}\left(S_{T}\right)\right\} \text { for } i=1, \ldots, m
$$

\section{Proof:}

Let us fix $i \in\{1, \ldots, m\}$ and denote $x_{i} \stackrel{\text { def }}{=} w_{0}^{i}+\mathbb{E}\left[e_{i}\left(S_{T}\right) \mathcal{K}_{\pi}\left(S_{T}\right)\right]$. Since $x_{i}>0$ we may find in view of (A1), (A2) some $y_{i}>0$ with $g\left(y_{i}\right)=x_{i}$.

Let $c\left(S_{T}\right)$ be a nonnegative random variable with $\mathbb{E}\left[c\left(S_{T}\right) \mathcal{K}_{\pi}\left(S_{T}\right)\right] \leq x_{i}$. Then

$$
\begin{aligned}
& \mathbb{E}\left[u\left\{S_{T}, c\left(S_{t}\right)\right\}\right]+y_{i}\left(x_{i}-\mathbb{E}\left[c\left(S_{T}\right) \mathcal{K}_{\pi}\left(S_{T}\right)\right]=y_{i} x_{i}+\mathbb{E}\left[u\left\{S_{T}, c\left(S_{t}\right)\right\}-y_{i} c\left(S_{T}\right) \mathcal{K}_{\pi}\left(S_{T}\right)\right] \leq\right. \\
& y_{i} x_{i}+\sup _{x \geq 0} \mathbb{E}\left[u\left(S_{T}, x\right)-y_{i} x \mathcal{K}_{\pi}\left(S_{T}\right)\right]= \\
& y_{i} x_{i}+\mathbb{E}\left[u\left[S_{T}, I_{i}\left\{S_{T}, y_{i} \mathcal{K}_{\pi}\left(S_{T}\right)\right\}\right]-y_{i} I_{i}\left\{S_{T}, y_{i} \mathcal{K}_{\pi}\left(S_{T}\right)\right\} \mathcal{K}_{\pi}\left(S_{T}\right)\right]=\mathbb{E}\left[u\left\{S_{T}, I_{i}\left(S_{T}, y_{i} \mathcal{K}_{\pi}\left(S_{T}\right)\right)\right\}\right] .
\end{aligned}
$$

Therefore $I_{i}\left(S_{T}, y_{i} \mathcal{K}_{\pi}\left(S_{T}\right)\right)$ solves the optimization problem of consumer $i$. Moreover, the numerical representation $U_{i}$ of consumer's $i$ preferences is strictly concave in view of strict concavity of $u^{i}(x, \cdot)$ for every $x \geq 0$. In particular $I_{i}\left(S_{T}, y_{i} \mathcal{K}_{\pi}\left(S_{T}\right)\right)$ is the unique solution, hence being identical with $\bar{c}_{i}\left(S_{T}\right)$.

Before starting with the proof of Theorem 3.1 let us consider for purposes of reference the classical case of the consumer being expected utility maximizer. Indeed as an additional corollary of Theorem 3.1, we may retain the folk result concerning the risk neutral price valuation and the v. Neumann-Morgenstern utility index of the representative agent. More precisely, let us assume that there exist mappings $u_{1}, \ldots, u_{r}$ from $\mathbb{R}_{+}$into $\mathbb{R} \cup\{-\infty\}$ satisfying $u^{1}(x, \cdot)=u_{1}, \ldots, u^{m}(x, \cdot)=u_{m}$ for $x \geq 0$, and

(A3) $u_{1}(y), \ldots, u_{m}(y) \in \mathbb{R}$ for $y>0$,

(A4) $u_{1}, \ldots, u_{m}$ are continuous, strictly increasing as well as strictly concave. 
Then

$$
u(y) \stackrel{\text { def }}{=} \sup \left\{\sum_{i=1}^{m} \alpha_{i} u_{i}\left(y_{i}\right) \mid y_{1}, \ldots, y_{m} \geq 0, \sum_{i=1}^{m} y_{i} \leq y\right\}=u_{\alpha}(x, y) \text { for } x, y \geq 0 .
$$

The adaptions of (10), (11) read as follows. We shall impose the so called Inada conditions on the state independent utility indices $u_{1}, \ldots, u_{m}$, i.e.

(A5) $\left.u_{1} \mid\right] 0, \infty\left[, \ldots, u_{m} \mid\right] 0, \infty[$ are assumed to be continuously differentiable satisfying

$$
\left.\lim _{e \rightarrow 0} \frac{d u_{i}}{d y}\right|_{y=e}=\infty,\left.\lim _{e \rightarrow \infty} \frac{d u_{i}}{d y}\right|_{y=e}=0(i=1, \ldots, m) .
$$

(A6) $\mathbb{E}\left[I_{1}\left(y \mathcal{K}_{\pi}\left(S_{T}\right)\right)\right], \ldots, \mathbb{E}\left[I_{m}\left(y \mathcal{K}_{\pi}\left(S_{T}\right)\right)\right]<\infty$ for any $y>0$, where $I_{1}, \ldots, I_{r}$ denote the inverses of $\frac{d u_{1}}{d y}, \ldots, \frac{d u_{m}}{d y}$ respectively.

We may conclude immediately from Theorem 3.1 the announced result.

Corollary A.2 Let $\bar{e}\left(S_{T}\right)=\frac{S_{T}}{S_{0}}$, and let $\left.u_{1} \mid\right] 0, \infty\left[, \ldots, u_{m} \mid\right] 0, \infty[$ be twice continuously differentiable. Then under (A3) - (A6), ul]0, $\infty\left[\right.$ is continuously differentiable, and for any $\alpha_{i}>0$ there exists some $\beta_{i}>0$ such that

$$
\left.\frac{d u}{d y}\right|_{y=\frac{s_{T}}{S_{0}}}=\left.\alpha_{i} \frac{d u_{i}}{d y}\right|_{y=\bar{c}_{i}\left(s_{T}\right)}=\alpha_{i} \beta_{i} \mathcal{K}_{\pi}\left(s_{T}\right) \text { for any realization } s_{T} \text { of } S_{T} .
$$

\section{Proof of Theorem 3.1:}

Without loss of generality let us set $\{1, \ldots, r\} \stackrel{\text { def }}{=}\left\{i \in\{1, \ldots, m\} \mid \alpha_{i}>0\right\}$. Then, defining $g_{i} \stackrel{\text { def }}{=} \alpha_{i} u_{i}$, we have $u_{\alpha}=\sum_{i=1}^{r} g_{i}$, and we may apply Lemmata B.1, B.2 and Proposition B.3 (cf. Appendix B). Then, in view of Lemmata B.1, B.2 and (3B), we obtain

$$
u_{\alpha}\left(s_{T}, \bar{e}\left[s_{T}\right]\right)=\sum_{i=1}^{r} \alpha_{i} u^{i}\left(s_{T}, \bar{c}^{i}\left[s_{T}\right]\right)
$$

for every realization $s_{T}$ of $S_{T}$.

On one hand by Theorem A.1, there exist $y_{1}, \ldots, y_{m}>0$ such that

$$
\bar{c}_{i}\left(S_{T}\right)=I_{i}\left(S_{T}, y_{i} \mathcal{K}_{\pi}\left(S_{T}\right)\right)>0 \text { for } i=1, \ldots, r .
$$

On the other hand, due to Proposition B.3, $\left.u_{\alpha}\left(s_{T}, \cdot\right) \mid\right] 0, \infty\left[\right.$ is differentiable for every realization $s_{T}$, satisfying

$$
\left.\alpha_{i} \frac{u^{i}\left(s_{T}, \cdot\right)}{d y}\right|_{y=\bar{c}^{i}\left(s_{T}\right)}=\left.\frac{u\left(s_{T}, \cdot\right)}{d y}\right|_{y=\bar{e}\left(s_{T}\right)}
$$

for $i \in\{1, \ldots, r\}$ and any realization $s_{T}$. Notice that by construction the random variable $\bar{e}\left(S_{T}\right)$ has strictly positive outcomes only. Now, the statement of Theorem 3.1 is clear. 


\section{B Appendix}

Throughout this section let the mappings $g_{1}, \ldots g_{r}: \mathbb{R}_{+}^{2} \rightarrow \mathbb{R} \cup\{-\infty\}$ satisfy the following conditions:

(B0) $g_{1}(x, y), \ldots, g_{r}(x, y) \in \mathbb{R}$ for $x \geq 0, y>0$;

(B1) $g_{1}(x, \cdot), \ldots, g_{r}(x, \cdot)$ are continuous, strictly increasing and strictly concave for $x \geq 0$;

(B2) $g_{1}(\cdot, y), \ldots, g_{r}(\cdot, y)$ are Borel-measurable for $y \geq 0$.

Furthermore let $g: \mathbb{R}_{+}^{2} \rightarrow \mathbb{R} \cup\{-\infty, \infty\}$ be defined by

$$
g(x, y)=\sup \left\{\sum_{i=1}^{r} g_{i}\left(x, y_{i}\right) \mid y_{1}, \ldots, y_{r} \geq 0, \sum_{i=1}^{r} y_{i} \leq y\right\} .
$$

Indeed $g(x, 0)=\sum_{i=1}^{r} g_{i}(x, 0) \in \mathbb{R} \cup\{-$ infty $\}$ for $x \geq 0$, and

$$
-\infty<\sum_{i=1}^{r} g_{i}\left(x, \frac{y}{r}\right) \leq g(x, y) \leq \sum_{i=1}^{r} g_{i}(x, y)<\infty
$$

for $x \geq 0, y>0$ due to (B0), (B1).

Lemma B.1 For any $x, y \geq 0$ there is some unique $\phi(x, y)=\left(\phi_{1}(x, y), \ldots, \phi(x, y)\right) \in \mathbb{R}_{+}^{m}$ such that $\sum_{i=1}^{m} \phi_{i}(x, y) \leq y$ and

$$
\sum_{i=1}^{r} g_{i}\left(x, \phi_{i}(x, y)\right)=g(x, y)
$$

Furthermore $\sum_{i=1}^{m} \phi_{i}(x, y)=y$.

\section{Proof:}

Let $x, y \geq 0$. For $y=0$ the statement of Lemma B.1 is obvious. So let $y>0$, which means $g(x, y) \in \mathbb{R}$.

Due to (B1), the mapping

$$
f:\left\{\left(y_{1}, \ldots, y_{r}\right) \in \mathbb{R}_{+}^{r} \mid \sum_{i=1}^{r} y_{i} \leq y, \sum_{i=1}^{r} g_{i}\left(x, y_{i}\right) \geq g(x, y)-1\right\} \rightarrow \mathbb{R},\left(y_{1}, \ldots, y_{r}\right) \mapsto \sum_{i=1}^{r} g_{i}\left(x, y_{i}\right)
$$

is continuous, strictly concave, and defined on a nonvoid convex compact set. Therefore $f$ attains it maximum at a unique $\phi(x, y)$. Obviously, $\sum_{i=1}^{r} \phi_{i}(x, y)=y$ because $f$ is strictly increasing too by (B1). The proof is complete.

Lemma B.1 defines a mapping $\phi=\left(\phi_{1}, \ldots, \phi_{r}\right): \mathbb{R}_{+}^{2} \rightarrow \mathbb{R}_{+}^{r}$. It is Borel-measurable as will be shown now. 
Lemma B.2 $\phi$ is Borel-measurable.

\section{Proof:}

It suffices to show that $\phi^{-1}\left(\underset{i=1}{\stackrel{r}{X}}\left[0, a_{i}\right]\right)$ is a Borel-subset of $\mathbb{R}_{+}^{2}$. For this purpose define for any $\left(a_{1}, \ldots, a_{r}\right)$ from $\mathbb{R}_{+}^{r}$ the mapping $g_{a_{1} \ldots a_{r}}: \mathbb{R}_{+} \times \mathbb{R}_{+} \rightarrow \mathbb{R} \cup\{-\infty\}$ by

$$
g_{a_{1} \ldots a_{r}}(x, y)=\sup \left\{\sum_{i=1}^{r} g_{i}\left(x, y_{i}\right) \mid\left(y_{1}, \ldots, y_{r}\right) \in \underset{i=1}{\stackrel{r}{X}}\left[0, a_{i}\right], \sum_{i=1}^{r} y_{i} \leq y\right\} .
$$

Notice that $g_{a_{1} \ldots a_{r}}(x, y) \in \mathbb{R}$ for $x \geq 0, y>0$, analogously to $g(x, y) \in \mathbb{R}$ for $x \geq 0, y>0$. Furthermore $g_{1}(x, \cdot), \ldots, g_{r}(x, \cdot)$ are continuous for any $x \geq 0$. Hence, setting $\mathcal{R}_{a_{1} \ldots a_{r}}=\stackrel{x}{X}_{i=1}^{r}\left[0, a_{i}\right] \times \mathbb{Q}^{m}$,

$$
g_{a_{1} \ldots a_{r}}^{-1}(] z, \infty[)=\bigcup_{\left(y_{1}, \ldots, y_{r}\right) \in \mathcal{R}_{a_{1} \ldots a_{r}}}\left(\sum_{i=1}^{r} \alpha_{i} g_{i}\left(\cdot, y_{i}\right)\right)^{-1}(] z, \infty[) \times\left[\sum_{i=1}^{r} y_{i}, \infty[(z \in \mathbb{R}) .\right.
$$

Thus $g_{a_{1} \ldots a_{r}}^{-1}(] z, \infty[)$ is a Borel-subset of $\mathbb{R}_{+}^{2}$ for every $z \in \mathbb{R}$ by assumption (B2). Then we may conclude that

$$
\phi^{-1}\left({\underset{i=1}{r}}_{i=1}\left[0, a_{i}\right]\right)=\left(\sup _{\left(b_{1}, \ldots, b_{r}\right) \in \mathbb{Q}_{+}^{r}} g_{b_{1} \ldots b_{r}}-g_{a_{1} \ldots a_{r}}\right)^{-1}(\{0\})
$$

is a Borel subset of $\mathbb{R}_{+}^{2}$ for any $\left(a_{1}, \ldots, a_{r}\right) \in \mathbb{R}_{+}^{r}$, which completes the proof.

In order to characterize the mapping $\phi$ in terms of derivatives of the functions $g_{1}(x, \cdot), \ldots, g_{r}(x, \cdot)$, it is customary to impose the Inada conditions, i.e.

(B3) for any $x \geq 0$ the mappings $\left.g_{1}(x, \cdot) \mid\right] 0, \infty\left[, \ldots, g_{r}(x, \cdot) \mid\right] 0, \infty[$ are assumed to be continuously differentiable satisfying

$$
\left.\lim _{\epsilon \rightarrow 0} \frac{\partial g^{i}(x, \cdot)}{\partial y}\right|_{y=\epsilon}=\infty,\left.\lim _{\epsilon \rightarrow \infty} \frac{\partial g_{i}(x, \cdot)}{\partial y}\right|_{y=e}=0, i=1, \ldots, r
$$

The Inada conditions together with condition (B1) imply that for any $i \in\{1, \ldots, r\}$ and every $x \geq 0$ the mapping $\left.\frac{\partial g_{i}(x, \cdot)}{\partial y} \mid\right] 0, \infty[$ is injective onto $] 0, \infty[$ with continuously differentiable, strictly decreasing inverse say $I_{i}(x, \cdot)$.

Proposition B.3 Let the assumptions (B0) - (B3) be fulfilled, and let $\left.g_{1}(x, \cdot) \mid\right] 0, \infty\left[, \ldots, g_{r}(x, \cdot) \mid\right] 0, \infty[$ be twice continuously differentiable.

Then for any $x \geq 0$ the mapping $g(x, \cdot) \mid] 0, \infty[$ is differentiable satisfying

$$
\phi(x, y)=\left(I_{1}\left[x,\left.\frac{\partial g(x, \cdot)}{\partial y}\right|_{y}\right], \ldots, I_{r}\left[x,\left.\frac{\partial g(x, \cdot)}{\partial y}\right|_{y}\right]\right) \text { for } y>0 .
$$




\section{Proof:}

Let for $x \geq 0$ the mapping $\left.F_{x}:\right] 0, \infty[\times] 0, \infty\left[\rightarrow \mathbb{R}\right.$ be defined by $F_{x}(y, z)=\sum_{i=1}^{r} I_{i}(x, z)-y$.

Since the mappings $\left.g_{1}(x, \cdot) \mid\right] 0, \infty\left[, \ldots, g_{r}(x, \cdot) \mid\right] 0, \infty[$ are assumed to be strictly concave and twice continuously differentiable, their second derivatives are strictly negative. Then by local inverse theorem the mappings $I_{1}(x, \cdot), \ldots, I_{r}(x, \cdot)$ are continuously differentiable, having strictly negative derivatives. In particular $F_{x}$ is continuously differentiable, satisfying

$$
\left.\frac{\partial F_{x}}{\partial z}\right|_{(y, z)} \neq 0 \text { for } y, z>0
$$

Furthermore, since $I_{1}(x, \cdot), \ldots, I_{r}(x, \cdot)$ are continuous and strictly decreasing mappings onto $] 0, \infty[$, we may find for any $y>0$ a unique $\varphi(y)>0$ with $F(y, \varphi(y))=0$. Drawing on the implicit function theorem, $y \mapsto \varphi(y)$ defines a differentiable mapping $\varphi:] 0, \infty[\rightarrow] 0, \infty[$.

Moreover, for $y>0$ and $y_{1}, \ldots, y_{r} \geq 0$ with $\sum_{i=1}^{r} y_{i} \leq y$, we may conclude

$$
\begin{aligned}
& \sum_{i=1}^{r} g_{i}\left(x, y_{i}\right)+\varphi(y)\left(y-\sum_{i=1}^{r} y_{i}\right)= \varphi(y) y+\sum_{i=1}^{r}\left(g_{i}\left(x, y_{i}\right)+\varphi(y) y_{i}\right) \leq \\
& \varphi(y) y+\sum_{i=1}^{r} \sup _{z \geq 0}\left(g_{i}(x, z)+\varphi(y) z\right)= \\
& \varphi(y) y+\sum_{i=1}^{r}\left[g_{i}\left\{x, I_{i}(x, \varphi(y))\right\}+\varphi(y) I_{i}\{x, \varphi(y)\}\right]= \\
& \sum_{i=1}^{r} g_{i}\left[x, I_{i}\{x, \varphi(y)\}\right]-F_{x}\{y, \varphi(y)\}=\sum_{i=1}^{r} g_{i}\left[x, I_{i}\{x, \varphi(y)\}\right] .
\end{aligned}
$$

This means

$$
g(x, y)=\sum_{i=1}^{r} g_{i}\left[x, I_{i}\{x, \varphi(y)\}\right]
$$

and hence by Lemma B.1

$\left(^{*}\right) \phi(x, y)=\left(I_{1}[x, \varphi(y)], \ldots, I_{r}[x, \varphi(y)]\right)$.

As a further consequence $g(x, \cdot) \mid] 0, \infty[$ is differentiable satisfying

$$
\left.\frac{d g(x, \cdot)}{d y}\right|_{y}=\left.\sum_{i=1}^{r} \varphi(y) \frac{d I_{i}(x, \cdot) \circ \varphi}{d y}\right|_{y}=\left.\varphi(y) \frac{d\left(\sum_{i=1}^{r} I_{i}(x, \cdot) \circ \varphi\right)}{d y}\right|_{y}=\varphi(y) .
$$

For the last equation notice that $\sum_{i=1}^{r} I_{i}(x, \cdot) \circ \varphi$ is just the identity on $] 0, \infty\left[\right.$. In view of $\left(^{*}\right)$ the proof is complete. 


\section{Appendix}

Proposition C.1 Let $g:] 0, \infty[\rightarrow \mathbb{R}$ be a mapping whose set of continuity points $\mathcal{C}(g)$ is an open subset of $\mathbb{R}$. Furthermore, let $F$ be an atomless distribution with satisfying $F([\underline{z}, \infty[)=1$ for some $\underline{z} \geq 0$. Additionally, let $\left(X_{N}\right)_{N}$ denote an i.i.d. sequence of random variables over some probability space $(\widetilde{\Omega}, \widetilde{\mathcal{F}}, \widetilde{\mathrm{P}})$ with common distribution $F$, inducing for any $N$ the order statistics $X_{1: N}, \ldots, X_{N: N}$ with $\underline{z} \stackrel{\text { def }}{=} X_{0: N} \leq X_{1: N} \leq$ $\ldots \leq X_{N: N}$. Then for any continuity point $x$ of $g$ which belongs to the topological interior int $(\operatorname{supp}(F))$ of the support of $F$ we obtain

$$
\sum_{i=1}^{N} g\left(X_{i: N}\right) \mathbf{1}_{] X_{(i-1): N}, X_{i: N}\right]} \rightarrow g(x) \text { a.s.. }
$$

In particular, setting $i(F) \stackrel{\text { def }}{=} \inf \{x \mid F(]-\infty, x])>0\}$ and $s(F) \stackrel{\text { def }}{=} \sup \{x \mid F(]-\infty, x])<1\}$, we may even achieve this convergence for any $x \in \mathcal{C}(g) \cap i(F), s(F)[$ if $\operatorname{supp}(F)$ is konvex.

\section{Proof:}

Let $x \in \mathcal{C}(g) \cap \operatorname{int}(\operatorname{supp}(F))$, let $q_{F}^{-}, q_{F}^{+}$denote the lower and upper quantile functions of $F$ respectively, and let us define $\left.\left.\alpha_{0} \stackrel{\text { def }}{=} F(]-\infty, x\right]\right)$. Since $F$ is atomless, and since $x \in \operatorname{int}(\operatorname{supp}(F))$ we have $\left.\alpha_{0} \in\right] 0,1[$ and $q_{F}^{-}\left(\alpha_{0}\right)=q_{F}^{+}\left(\alpha_{0}\right)=x$.

Let $\varepsilon>0$ be fixed. By assumption $\mathcal{C}(g)$ is open so that there is some $\delta>0$ such that $g \mid] x-\delta, x+\delta[$ is continuous, and $|g(y)-g(x)|<\varepsilon$ for any $y \in] x-\delta, x+\delta[$. Furthermore, the lower and upper quantile functions of $F$ differ on at most countable points only, and the distribution function of $F$ is continuous. Hence we may find an antitone sequence $\left(\alpha_{n}\right)_{n \in \mathbb{N}}$ in $] \alpha_{0}, 1\left[\right.$ which converges to $\alpha_{0}$ such that $q_{F}^{-}\left(\alpha_{n}\right)=$ $q_{F}^{+}\left(\alpha_{n}\right) \stackrel{\text { def }}{=} q_{F}\left(\alpha_{n}\right)$ for every $n \in \mathbb{N}$.

We may draw on the asympotic theory of order statistics to find some $A \in \widetilde{\mathcal{F}}, \widetilde{\mathrm{P}}(A)=1$, such that

$$
\lim _{N \rightarrow \infty} X_{1: N}(\omega)=i(F) \text { for } \omega \in A, \quad \text { and } \lim _{N \rightarrow \infty} X_{\left[N \alpha_{j}+1\right]: N}(\omega)=q_{F}\left(\alpha_{j}\right)
$$

holds for every $\omega \in A$ and any $j \in \mathbb{N}$, where $\left[N \alpha_{j}+1\right]$ denotes the largest $l \in \mathbb{N}$ with $l \leq N \alpha_{j}+1$ (cf. Witting and Müller-Funk (1995), Satz 7.108, Satz 7.120).

Now let us fix $\omega \in A$. Putting things together, we have $\left.q_{F}\left(\alpha_{j}\right) \in\right] x, x+\delta[$, and thus

$$
X_{1: N}(\omega)<x<X_{\left[N \alpha_{j}+1\right]: N}(\omega)<x+\delta
$$


for sufficiently large $j$ and $N$. Hence, setting $i_{N} \stackrel{\text { def }}{=} \min \left\{i \in\{1, \ldots, N\} \mid x \leq X_{i: N}(\omega)\right\}$, we obtain the inequalities $X_{i_{N}: N}(\omega) \leq X_{\left[N \alpha_{j}+\right]: N}(\omega)<x+\delta$, implying

$$
\left|\sum_{i=1}^{N} g\left(X_{i: N}\right) \mathbf{1}_{\left.X_{(i-1): N}, X_{i: N}\right]}(\omega)-g(x)\right|=\left|g\left(X_{i_{N}: N}(\omega)\right)-g(x)\right|<\varepsilon .
$$

The remaining part of follows immediately from the observation that $\operatorname{int}(\operatorname{supp}(F))=] i(F), s(F)[$ is valid for convex $\operatorname{supp}(F)$.

\section{References}

Ait-Sahalia, Y. and Lo, A. (2000). Nonparametric risk-management and implied risk aversion. Journal of Econometrics, 94(9):9-51.

Bauer, H. (1992). Mass- und Integrationstheorie. de Gruyter, Berlin, 2nd edition.

Billingsley, P. and Topsoe, F. (1967). Uniformity in weak convergence. Z. Wahrscheinlichkeitstheorie verw. Geb., 7:1-16.

Chabi-Yo, Y., Garcia, R., and Renault, E. (2008). State dependence can explain the risk aversion puzzle. Review of Financial Studies, 21:973-1011.

Constantinidis, G. M., Jackwerth, J. C., and Perrakis, S. (2009). Mispricing of S\&P 500 Index Options. Forthcoming in Review of Financial Studies.

DalMaso, G. (1993). An Introduction to $\Gamma$-Convergence. Birkhäuser, Boston et al.

Dana, R.-A. and Jeanblanc, M. (2003). Financial Markets in Continuous Time. Springer, Berlin.

Detlefsen, K., Härdle, W. K., and Moro, R. A. (2007). Empirical pricing kernels and investor preferences. SFB 649 Discussion Paper 017, Humboldt-Universität zu Berlin.

Duffie, D. (1996). Dynamic Asset Pricing Theory, 2nd ed. Princeton University Press.

Dunford, N. and Schwarz, J. (1958). Linear Operators. Part I. Interpublisher Sciences, New York. 
Golubev, Y., Härdle, W. K., and Timofeev, R. (2008). Testing monotonicity of pricing kernels. SFB 649 Discussion Paper 001, Humboldt-Universität zu Berlin.

Jackwerth, J. (2000). Recovering risk aversion from option prices and realized returns. Review of Financial Studies, 13(2):433-451.

Karatzas, I. and Shreve, S. E. (1998). Methods of Mathematical Finance. Springer, New York.

Karatzas, I. and Zitkovic, G. (2003). Optimal consumption from investment and random endowment in incomplete semimartingale markets. The Annals of Probability, 31:1821-1858.

Karni, E., Schmeidler, D., and Vind, K. (1983). On state dependent preferences and subjective probabilities. Econometrica, 51(4):1021-1031.

Kramkov, D. and Schachermayer, W. (1999). The asymptotic elasticity of utility functions and optimal investment in incomplete markets. The Annals of Applied Probability, 9(3):904-950.

Mas-Colell, A., Whinston, M. D., and Greene, J. R. (1995). Microeconomic Theory. Oxford University Press.

Witting, H. and Müller-Funk, U. (1995). Mathematische Statistik II. Teubner, Stuttgart. 


\section{SFB 649 Discussion Paper Series 2009}

For a complete list of Discussion Papers published by the SFB 649, please visit http://sfb649. wiwi. hu-berlin.de.

001 "Implied Market Price of Weather Risk" by Wolfgang Härdle and Brenda López Cabrera, J anuary 2009.

002 "On the Systemic Nature of Weather Risk" by Guenther Filler, Martin Odening, Ostap Okhrin and Wei Xu, January 2009.

003 "Localized Realized Volatility Modelling" by Ying Chen, Wolfgang Karl Härdle and Uta Pigorsch, January 2009.

004 "New recipes for estimating default intensities" by Alexander Baranovski, Carsten von Lieres and André Wilch, January 2009.

005 "Panel Cointegration Testing in the Presence of a Time Trend" by Bernd Droge and Deniz Dilan Karaman Örsal, J anuary 2009.

006 "Regulatory Risk under Optimal Incentive Regulation" by Roland Strausz, January 2009.

007 "Combination of multivariate volatility forecasts" by Alessandra Amendola and Giuseppe Storti, January 2009.

008 "Mortality modeling: Lee-Carter and the macroeconomy" by Katja Hanewald, January 2009.

009 "Stochastic Population Forecast for Germany and its Consequence for the German Pension System" by Wolfgang Härdle and Alena Mysickova, February 2009.

010 "A Microeconomic Explanation of the EPK Paradox" by Wolfgang Härdle, Volker Krätschmer and Rouslan Moro, February 2009. 